\title{
Changes and sex- and age-related differences in the expression of drug metabolizing enzymes in a KRAS-mutant mouse model of lung cancer
}

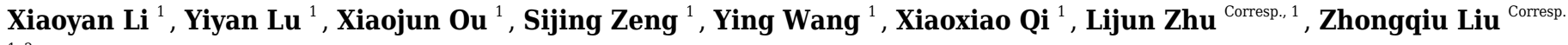 \\ 1,2 \\ 1 International Institute for Translational Chinese Medicine, Guangzhou University of Chinese Medicine, guangzhou, China \\ 2 State Key Laboratory of Quality Research in Chinese Medicine, Macau University of Science and Technology, Macau, China \\ Corresponding Authors: Lijun Zhu, Zhongqiu Liu \\ Email address: zhulijun@gzucm.edu.cn, liuzq@gzucm.edu.com
}

Background: This study aimed to systematically profile the alterations and sex- and age-related differences in the drug metabolizing enzymes (DMEs) in a KRAS-mutant mouse model of lung cancer (KRAS mice). Methodology: In this study, the LC-MS/MS approach and a probe substrate method were used to detect the alterations in 21 isoforms of DMEs, as well as the enzymatic activities of five isoforms, respectively. Western blotting was applied to study the protein expression of four related receptors.

Results: The proteins contents of CYP2C29 and CYP3A11, were significantly downregulated in the livers of male KRAS mice at 26 weeks (3.7- and 4.4-fold, respectively, $p<0.05)$. SULT1A1 and SULT1D1 were upregulated by 1.8- to 7.0- fold at 20 ( $p=0.015$ and 0.017 , respectively) and 26 weeks ( $p=0.055$ and 0.031 , respectively). There were positive correlations between protein expression and enzyme activity for CYP2E1, UGT1A9, SULT1A1 and SULT1D1 $\left(r^{2} \geq 0.5, p<0.001\right)$. Western blotting analysis revealed the downregulation of AHR, FXR and PPAR $\alpha$ protein expression in male KRAS mice at 26 weeks. For sexrelated differences, CYP2E1 was male-predominant and UGT1A2 was female-predominant in the kidney. UGT1A1 and UGT1A5 expression was female-predominant, whereas UGT2B1 exhibited male-predominant expression in liver tissue. For the tissue distribution of DMEs, 21 subtypes of DMEs were all expressed in liver tissue. In the intestine, the expression levels of CYP2C29, CYP27A1, UGT1A2, 1A5, 1A6a, 1A9, 2B1, $2 \mathrm{~B} 5$ and $2 \mathrm{~B} 36$ were under the limitation of quantification. The subtypes of CYP7A1, 1B1, 2E1 and UGT1A1, 2A3, 2B34 were detected in kidney tissue. Conclusions: This study, for the first time, unveils the variations and sex- and age-related differences in DMEs in C57 BL/6 (WT) mice and KRAS mice. 
1 TITLE PAGE

2

3 Changes and sex- and age-related differences in the expression of drug metabolizing

4 enzymes in a KRAS-mutant mouse model of lung cancer

5

6 Xiaoyan $\mathrm{Li}^{1}$, Yiyan $\mathrm{Lu}^{1}$, Xiaojun $\mathrm{Ou}^{1}$, Sijing Zeng ${ }^{1}$, Ying Wang ${ }^{1}$, Xiaoxiao $\mathrm{Qi}^{1}$, Lijun Zhu ${ }^{{ }^{*}}$,

$7 \quad$ Zhongqiu Liu $^{1,2 *}$

8

$9{ }^{1}$ International Institute for Translational Chinese Medicine, Guangzhou University of Chinese

10 Medicine, Guangzhou, Guangdong, 510006, China.

$11{ }^{2}$ State Key Laboratory of Quality Research in Chinese Medicine, Macau University of Science

12 and Technology, Macau (SAR), China.

13

14 *Address correspondence to:

15 Prof. Zhongqiu Liu, International Institute for Translational Chinese Medicine, Guangzhou

16 University of Chinese Medicine, Guangzhou, Guangdong, 510006, PR China. Tel: +8620-

17 39358061; Fax: +8620-39358071; E-mail: liuzq@gzucm.edu.cn.

18

19 Dr. Lijun Zhu, International Institute for Translational Chinese Medicine, Guangzhou,

20 Guangdong, 510006, PR China. Tel: +8620-39358401; Fax: +8620-39358071; E-mail:

21 zhulijun@gzucm.edu.cn. 
22 ABSTRACT

23 Background: This study aimed to systematically profile the alterations and sex- and age-related

24 differences in the drug metabolizing enzymes (DMEs) in a KRAS-mutant mouse model of lung

25 cancer (KRAS mice).

26 Methodology: In this study, the LC-MS/MS approach and a probe substrate method were used to

27 detect the alterations in 21 isoforms of DMEs, as well as the enzymatic activities of five

28 isoforms, respectively. Western blotting was applied to study the protein expression of four

29 related receptors.

30 Results: The proteins contents of CYP2C29 and CYP3A11, were significantly downregulated in

31 the livers of male KRAS mice at 26 weeks (3.7- and 4.4-fold, respectively, $p<0.05$ ). SULT1A1

32 and SULT1D1 were upregulated by 1.8- to 7.0- fold at 20 ( $p=0.015$ and 0.017 , respectively) and

3326 weeks ( $p=0.055$ and 0.031 , respectively). There were positive correlations between protein

34 expression and enzyme activity for CYP2E1, UGT1A9, SULT1A1 and SULT1D1 $\left(\mathrm{r}^{2} \geq 0.5, p<\right.$

35 0.001). Western blotting analysis revealed the downregulation of AHR, FXR and PPAR $\alpha$ protein

36 expression in male KRAS mice at 26 weeks. For sex-related differences, CYP2E1 was male-

37 predominant and UGT1A2 was female-predominant in the kidney. UGT1A1 and UGT1A5

38 expression was female-predominant, whereas UGT2B1 exhibited male-predominant expression

39 in liver tissue. For the tissue distribution of DMEs, 21 subtypes of DMEs were all expressed in

40 liver tissue. In the intestine, the expression levels of CYP2C29, CYP27A1, UGT1A2, 1A5, 1A6a,

41 1A9, 2B1, 2B5 and 2B36 were under the limitation of quantification. The subtypes of CYP7A1,

42 1B1, 2E1 and UGT1A1, 2A3, 2B34 were detected in kidney tissue. 
43 Conclusions: This study, for the first time, unveils the variations and sex- and age-related

44 differences in DMEs in C57 BL/6 (WT) mice and KRAS mice.

45 Keywords: Drug metabolizing enzyme (DME); KRAS mutant mouse model of lung cancer

46 (KRAS mice); Liquid chromatography tandem mass spectrometry (LC-MS/MS). 


\section{INTRODUCTION}

48 Many studies have posited that disease states, as well as sex- and age-related differences can

49 alter the expression of drug metabolizing enzymes (DMEs), and in turn change the metabolism

50 and detoxification of drugs by remodeling the hepatic absorption, distribution, metabolism and

51 excretion (ADME) (Court 2010; Hui Li et al. 2017; Beatrice A et al. 2014; S. Xu et al. 2019; Wu

52 and Lin 2018). Therefore, we specifically investigated the changes in DME expression levels in a

53 disease model with age- and sex-related differences.

54 The KRAS mutation, the most frequently mutated isoform of RAS, accounts for $>85 \%$ of

55 RAS-driven cancers (Ding et al. 2008; Nussinov et al. 2015). However, to date, it is still a major

56 challenge to develop novel drugs that effectively treat KRAS mutant lung cancer (Mccormick

57 2015). Considering the widespread and incurable nature of this disease, the metabolic profile of

58 drugs is urgently needed to determine when KRAS is mutated. The mouse genome is $99 \%$

59 identical to the human genome, and the organs and systemic physiology of mouse have similar

60 patterns with humans (Robert A. Ribeiro et al. 2006). Hence, mice have been widely used in

61 current cancer research ( 59\% of the total number of animals used) (Wang et al. 2020). In our

62 study, a KRAS-mutant mouse model of lung cancer (KRAS mice, spontaneous tumors in the

63 lung) was used to study the changes in DMEs in the development of lung cancer. KRAS mice

64 were first observed to have small pleural nodules at one week of age, and numerous pleural

65 lesions started to appear in 5-weeks-old mice (Leisa Johnson et al. 2001). Advanced tumours

66 begin to appear in the lung of KRAS mice at 20 weeks and its life span is approximately 28

67 weeks (Leisa Johnson et al. 2001). 
68 The activation and inactivation of exogenous drugs are mainly regulated by drug metabolizing

69 enzymes (DMEs), including cytochrome P450s (CYPs), UDP-glucuronosyltransferases (UGTs),

70 and sulfotransferases (SULTs) (T. Yan et al. 2014; Cong Xie et al. 2017). The changes in DMEs

71 can further affect the efficacy of the drug and even increase the side effects. For instance,

72 irinotecan, which is used in the treatment of metastatic colorectal cancer, causes severe intestinal

73 toxicity attributed to damage to UGT1A (Ronaldo A. Ribeiro et al. 2016). It was also reported

74 that CYP3A has higher expression in osteosarcoma (By H. R. Dhaini et al. 2003). The activation/

75 inactivation of anticancer drugs metabolized by CYP3A would lead to changes in curative effect.

76 Sex and age are important factors influencing the expression level of DMEs (Kennedy 2008; $\mathrm{H}$.

77 Zheng et al. 2018). For age-related differences, a related report showed that the enzyme activity

78 of UGT1A increases before 20 years of age and then decreases (Court 2010). Sex-related

79 differences characterize the metabolism of many drugs used frequently in the clinic (Waxman

80 and Holloway 2009). For instance, men showed a 38\% faster clearance of olanzapine than

81 women (Kristin L. Bigos et al. 2008). Therefore, a thorough understanding of the variations in

82 DMEs is beneficial and indispensable for pharmacological evaluation and rational clinical drug

83 use.

84 To effectively treat the KRAS-mutant lung cancer, researchers have used many drugs, such as

85 gefitinib, erlotinib, cisplatin, trametinib, and pazopanib (Min K. Kim et al. 2018; Jean L. K. Pujol

86 et al. 2006). Changes in DMEs could alter the metabolic characteristics of these drugs, further

87 affecting their efficacy in vivo. The drugs erlotinib and cisplatin are mainly metabolized by

88 CYP3A4, an ortholog of mouse CYP3A11 (Melanie et al. 2015; Hanna K. Sanoff et al. 2010).

89 Alterations in the activity of CYP3A4 could potentially have a pronounced effect on drug 
90 exposure. In other words, downregulation of CYP3A4 could reduce sorafenib hepatotoxicity (T.

91 Yan et al. 2015). However, little is known about the alterations in DMEs after KRAS mutation,

92 thus causing some difficulties in understanding the fate of drugs in vivo and leading to confusion

93 about the efficacy and side effects.

94 MS-based quantifications are different from traditional immunogenic methods and can

95 increase the sensitivity and high throughput of the absolute quantification of proteins. In our

96 study, an LC-MS/MS method was employed to determine the protein expression of DMEs in WT

97 and KRAS mice (J. Chen et al. 2017). In addition, the possible mechanism for the variations in

98 DMEs was investigated. We intend to provide a valuable reference for pharmacological

99 evaluation and rational clinical drug use in patients with KRAS mutated lung cancer. 


\section{MATERIALS AND METHODS}

101 Chemicals and Reagents. Ammonium bicarbonate (AB), dithiothreitol (DTT), iodoacetamide

102 (IAA), trifluoroacetic acid (TFA) and phenylmethanesulfonyl fluoride (PMSF) were bought from

103 Sigma-Aldrich, USA. Sequencing grade modified trypsin was obtained from Promega (Madison,

104 WI). All peptides and internal standard (purity > 95\%) were got from Your R\&D Partner. HPLC-

105 grade methanol, formic acid and acetonitrile were acquired from Merck (Darmstadt, Germany).

106 NADPH solution A and NADPH solution B were got from BD Bioscience, USA. Alamethicin,

107 Tetracycline, Glucosyl monophosphate, Uridine diphosphate glucuronic acid (UDPGA), 3 *

108 phosphoadenosine-5 * -phosphosulfate (PAPS), MgCl2, Chlorzoxazone, Testosterone, 6 $\beta$ -

109 hydroxytestosterone, Propofol, Dopamine, 6-hydroxy chlorzoxazone, Propofol-glucuronide and

110 4-Nitrophenyl sulfate metabolite were acquired from Sigma-Aldrich, USA. Dopamine 3-O-

111 sulfate and dopamine 4-O-sulfate were got from TRC, Canada. P-Nitrophenol was bought from

112 Aladdin, China. Genistein and ammonium acetate were got from Chengdu Mansite

113 Biotechnology Co., Ltd. and Dalian Meilun Biotechnology Co., Ltd., respectively. Coomassie

114 brilliant blue, providing for protein measurement, was bought from Bio-Rad (Hercules,

115 California, USA).

116 Animals. Male and female C57 BL/6 mice (5, 10, 15, 20, 26 weeks, n=5) were obtained from

117 Vital River Laboratory Animal Technology Co. Ltd (Beijing, China). Male and female B6.129S-

118 Kras $^{\mathrm{tm} 3 \mathrm{Tyj}} / \mathrm{Nci}$ K-ras ${ }^{\mathrm{LA2}}$ (5, 10, 15, 20, 26 weeks, n=5) were acquired from National Cancer

119 Insitute. The animals were kept at controlled temperature of $24-26{ }^{\circ} \mathrm{C}$ and humidity of $50-60 \%$,

120 with a $12 \mathrm{~h}$ light-dark cycle. The permission of all animal experiments was obtained from the 
121 Institutional Animal Care and Use Committee of the International Institute for Translational

122 Chinese Medicine (IITCM_20171105). Before the experiment, animals were fasted overnight but

123 allowed free access to water. All procedures were performed under diethyl ether anesthesia and

124 the efforts were made to minimize suffering. After the animal experiment was completed, the

125 animal bodies were frozen and sent to professionals for harmless treatment.

126 Histopathological analysis of lung tissues. Lung tissues were acquired from KRAS mice and

127 wild-type (WT) mice. The morphology of lung tissues was observed under stereoscopic

128 microscope (Leica, M165C), then tissues were fixed by 4\% paraformaldehyde. After

129 paraformaldehyde fixation and paraffin embedding, mouse lung tissues were sliced and stained

130 with hematoxylin for $30 \mathrm{~s}$ and $0.5 \%$ eosin for $10 \mathrm{~s}$, and covered with neutral gum. The images

131 were obtained under microscopy (NIKON Eclipseci, Japan).

132 Preparation of mouse S9 fractions. Mouse tissue (liver, kidney and intestine) were isolated

133 from WT and KRAS mice. The tissues were minced and washed with ice cold saline. Ice-cold

134 homogenization buffer (50 mM potassium phosphate, $250 \mathrm{mM}$ sucrose, $1 \mathrm{mM}$ EDTA, PH 7.4)

135 with $0.28 \mathrm{mM}$ phenylmethylsulfonyl fluoride (PMSF) was added to the minced tissues and

136 homogenized until an even suspension was obtained. Then the suspension was centrifuged at

$1379,000 \times \mathrm{g}$ for $20 \mathrm{~min}$ at $4^{\circ} \mathrm{C}$. The supernatant was collected as S9 fractions (L. Tang et al. 2012;

138 Zhu et al. 2010). Liver tissue was handled with a single sample of each mouse while kidney and

139 intestine tissues of each group of mice $(n=5)$ were mixed into same sample. Protein

140 concentrations of mouse S9 fractions were detected by coomassie brilliant blue and the bovine

141 serum albumin was selected as the standard.

142 LC-MS/MS analysis. Eight isoforms of CYPs (CYP1B1, CYP2C29, CYP2D22, CYP2E1, 
143 CYP3A11, CYP3A25, CYP7A1 and CYP27A1), ten isoforms of UGTs (UGT1A1, UGT1A2,

144 UGT1A5, UGT1A6a, UGT1A9, UGT2A3, UGT2B1, UGT2B5, UGT2B34 and UGT2B36) and

145 three isoforms of SULTs (SULT1A1, SULT1B1 and SULT1D1) were analyzed. The methods of

146 sample preparation and quantifying DMEs amounts by UHPLC/MS-MS were consistent with our

147 previous study and dynamic MRM chromatograms of 21 subtypes were displayed in

148 Supplemental Figure 1 (J. Chen et al. 2017). Samples were analyzed by using an Agilent 6490

149 triple quadruple mass spectrometer coupled with 1290 Infinite UHPLC system. A Poroshell C 18

150 column $(2.1 \mathrm{~mm} \times 100 \mathrm{~nm}, 2.7 \mu \mathrm{m}$; Agilent Technologies $)$ was used for separation. In this study,

151 the protein amounts of DMEs were represented in the form of pmol protein per S9 fraction

152 protein (pmol/mg). The quantification of protein levels was performed two independent experiments.

153 All samples were performed in triplicate in each independent experiment and data were presented

154 as mean \pm SD.

155 Enzyme assays of liver S9 fractions. Enzyme activities of CYP2E1, CYP3A11, UGT1A9,

156 SULT1A1 and SULT1D1 were measured by specific probe substrates in vitro (chlorzoxazone,

157 testosterone, propofol, p-nitrophenol and dopamine, respectively). The enzyme activities of these

158 isoforms in mice were determined by incubating S9 fractions with appropriate substrate

159 concentrations. Production of metabolites was quantified to value the activities of these isoforms

160 between WT and KRAS mice at their different age. The incubation systems of CYPs, UGTs and

161 SULTs, are in accordance with our previous articles with minor modification (C. Xie et al. 2017;

162 T. Yan et al. 2015; H. Zheng et al. 2018). In order to terminate the reaction, $200 \mu \mathrm{L}$ methanol

163 with $200 \mathrm{nM}$ genistein was added. Then, the solution was vortexed and thereafter centrifuged for

$16430 \mathrm{~min}$ at $18000 \mathrm{~g}$. Then the supernatant of all samples was injected to analyze by LC-MS/MS. 
165 The enzyme activity was measured from 2 independent experiments. Each sample was performed in

166 triplicates in each independent experiment and data was presented as mean \pm SD.

167 Western blotting. The protein levels of aryl hydrocarbon receptor (AHR), bile acid receptor

168 (FXR), pregnane X receptor (PXR) and peroxisome proliferator-activated receptor (PPAR $\alpha$ ) were

169 determined in male WT and KRAS mice at 26 weeks, and $\beta$-actin was used as an internal control.

170 The S9 samples were mixed with $5 \times$ loading buffer and the mixture was denatured at $100{ }^{\circ} \mathrm{C}$ for

$1715 \mathrm{~min}$. An equal amount of protein $(40 \mu \mathrm{g})$ was separated by SDS-PAGE at a voltage of $120 \mathrm{~V}$ to

172 the correct band size and the protein was subsequently transferred from the gel to the PVDF

173 membrane. Then, the membrane was blocked for $1 \mathrm{~h}$ with 5\% non-fat milk (w/v) in Tris-buffered

174 saline containing $0.1 \%$ Tween-20 (TBST). The corresponding primary antibodies, against mouse

175 peroxisome proliferator-activated receptor (PPAR $\alpha$, sc-398394), pregnane X receptor (PXR,

176 ab118336), bile acid receptor (FXR, ab28480) and aryl hydrocarbon receptor (AHR, ab2769) and

$177 \beta$-ACTIN (from Cell Signaling Technology, CST, Boston, USA) were diluted to a recommended

178 dilution of 1:1000 with 5\% non-fat milk according to the manufacturer's instructions. After

179 blocking, the membrane was incubated with the corresponding primary antibodies at $4{ }^{\circ} \mathrm{C}$

180 overnight with gentle shaking and was then washed before being incubated with the

181 corresponding secondary antibody at a dilution of 1:2000-1:3000 for $1 \mathrm{~h}$ at room temperature.

182 ECL chemiluminescence was used to detect the signals and each protein band was quantified by

183 Image J (National Institutes of Health, Hercules, CA, USA). The WB analysis was performed

184 from 2 independent experiments, and each target protein was analyzed twice in each independent

185 experiment. The data was presented as mean \pm SD.

186 Data analysis. One-way ANOVA analysis, non-parametric test and independent sample t tests 
187 were conducted using SPSS 19.0 to evaluate statistical differences. Correlation analyses were

188 performed using SPSS 19.0 and GraphPad Prism 7, according to the Pearson product-moment

189 correlation for normal related data and Spearman's rank correlation for non-normally related data.

190 Partial least squares discriminant analysis (PLS-DA) was performed to visualize the changes of

191 DMEs after KRAS mutation using SIMCA-P 14.0 tool (Umetrics, Umea, Sweden). In each case,

192 a value of $p<0.05$ denotes statistical significance for all of the statistical analyses. 


\section{RESULTS}

194 The phenotypic characteristics of KRAS mice. Pulmonary morphology was observed using

195 a stereoscope (Fig. 1A-B). Compared with those in the WT mice, many lung nodules were

196 observed in the lung tissues of the KRAS mice (Fig. 1B, As the arrows point), and the lung

197 tissues appeared dull overall. Histological and pathological features of the WT and KRAS mice

198 were detected by H\&E staining (Fig. 1C-H and Supplemental Fig. 2). The morphology of lung

199 cells in the WT mice was normal, whereas in the KRAS mice, the lung cells were

200 hyperproliferative (Fig. 1F-H and Supplemental Fig. 2, As the arrows point). The nuclei were

201 deeply stained and lager.

202 Alterations in the protein contents of DMEs by KRAS mutation. In male mice, PLS-DA

203 analysis was applied to evaluate the clustering between the male WT and KRAS mice based on

204 the expression of 21 DMEs (Fig. 2A-E). We observed obvious distinctions between the male WT

205 and KRAS mice at 5, 10, 15, 20 and 26 weeks. These results demonstrated the differences in

206 DMEs among them. As shown in Fig. 2H in liver tissue, SULT1A1 increased by 2.4-fold at 5

207 weeks ( $p=0.016)$; CYP27A1 and UGT1A1 increased by 1.9-fold and 2.3-fold at 15 weeks

208 respectively ( $p=0.001$ and $p>0.05$, respectively); SULT1A1 and SULT1D1 were upregulated

209 by 3.4-fold and 1.8-fold at 20 weeks, respectively ( $p=0.015$ and $p=0.017$, respectively); and at

21026 weeks, SULT1A1 and SULT1D1 were upregulated by 2.0-fold and 1.8-fold respectively ( $p>$

2110.05 and $p=0.031$, respectively), and CYP2C29, CYP3A11, CYP27A1 and UGT1A5 decreased

212 by 3.7-fold ( $p=0.005), 4.4$-fold $(p=0.004), 2.1$-fold $(p=0.043$ ) and 2.3-fold $(p=0.014)$,

213 respectively. In intestinal tissue, SULT1B1 and SULT1D1 increased by 3.2-fold and 2.9-fold at 
21420 weeks, respectively ( $p>0.05)$; and SULT1A1 and SULT1D1 increased by 2.0-fold $(p>0.05)$

215 and 1.8-fold at 26 weeks $(p=0.024)$, respectively. In kidney tissue, SULT1D1 was upregulated

216 by 3.0 -fold at 26 weeks $(p>0.05)$.

217 Supplemental Fig. 3 shows some changes in DMEs with KRAS mutations in female KRAS

218 mice. In liver tissue, UGT1A9 decreased by 2.3-fold at 5 weeks ( $p=0.002)$; UGT2B1 decreased

219 by 2.1-fold at 10 weeks ( $p=0.004)$; CYP2C29, CYP2D22, CYP2E1, CYP3A11, CYP27A1,

220 UGT1A1, UGT2A3, UGT2B5 and UGT2B1 were upregulated by 2.5- $(p=0.014), 1.7-(p=$

$2210.018), 2.8-(p=0.001), 2.6-(p=0.001), 1.8-(p=0.014), 2.5-(p=0.008), 2.2-(p=0.005), 2.3-$

222 fold $(p=0.014)$, and 2.4 folds $(p=0.002)$, respectively, at 15 weeks; SULT1A1 increased by 2.2

223 folds at 20 weeks ( $p>0.05)$; and CYP3A11 decreased by 2.1-fold at 26 weeks $(p=0.033)$. In

224 intestine tissue, SULT1B1 and SULT1D1 increased by 3.4-fold $(p>0.05)$ and 4.7-fold $(p=$

225 0.042), respectively, at 26 weeks. In kidney tissue, SULT1D1 increased by 1.9 folds at 26 weeks

$226(p=0.013)$.

227 Alterations in DME activities of DMEs by KRAS mutation. As shown in Fig. 3, the activity

228 of CYP3A11 was significantly downregulated in the male KRAS mice with aging. Furthermore,

229 there were significant differences between the WT and KRAS mice. UGT1A9 gradually declined

230 in the male mice from 5 to 26 weeks. SULT1A1 and SULT1D1 displayed larger differences at 20

231 and 26 weeks in the male KRAS mice than in the WT mice. SULT1A1 increased by 2.2- $(p>$

$2320.05)$ and 3.9-fold ( $p=0.008)$, respectively. SULT1D1 was upregulated by 7.0- $(p=0.007)$ and

233 3.5-fold ( $p>0.05$ ), respectively. Supplemental Fig. 4 shows the activities in the female WT and

234 KRAS mice at different ages. The activities of CYP2E1 and SULT1D1 displayed no significant

235 differences in the WT and KRAS mice with increasing age. CYP3A11 displayed an increasing 
236 tendency from 5 to 26 weeks. The activity of UGT1A9 showed a significant decrease at 15, 20

237 and 26 weeks compared to that at 5 weeks. At 15 weeks, the activity of SULT1A1 was markedly

238 different in the KRAS mice relative to the WT mice $(p=0.022)$.

239 Correlation of protein content and enzyme activity of DMEs. The enzyme activities of

240 CYP2E1, CYP3A11, UGT1A9, SULT1A1 and SULT1D1 were compared with their protein

241 contents. The correlation analysis assessed the protein levels quantified by LC-MS/MS and the

242 activities detected by the specific probes. As shown in Fig. 4, there was a good correlation

243 between enzyme activity and protein content (CYP2E1, $\mathrm{r}^{2}=0.54, p<0.001$; UGT1A9, $\mathrm{r}^{2}=0.55, p$

$244<0.001$; SULT1A1, $\mathrm{r}^{2}=0.62, p<0.001$; SULT1D1, $\left.\mathrm{r}^{2}=0.89, p<0.001\right)$. A poor correlation for

245 CYP3A11 was observed in the mouse liver $\left(\mathrm{r}^{2}<0.50, p>0.05\right)$.

246 Protein expression profiles of AHR, FXR, PPAR $\alpha$ (H-2) and PXR. To evaluate the protein

247 levels of receptors, we dissected liver tissue from the KRAS mice. As shown in Fig. 5, the protein

248 expression levels of AHR, FXR and PPAR $\alpha(\mathrm{H}-2)$ were downregulated by 40.22\%, 20.90\% and

$24926.76 \%$ in the livers of the male KRAS mice, respectively ( $p=0.000,0.035$ and 0.005 ,

250 respectively). Compared to the WT mice, the KRAS mice showed no significant difference in the

251 protein amount of PXR (decreased by 13.52\%, $p=0.109$ ). In the female mice (Supplemental Fig.

252 5), the protein expression levels of AHR, FXR, PPAR $\alpha(H-2)$ and PXR were downregulated by

$25312.00 \%, 10.14 \%, 5.60 \%$ and $38.06 \%$ in the liver, respectively $(p=0.466,0.442,0.710$ and 0.074 ,

254 respectively).

255 Tissue distribution of DMEs. To evaluate the tissue distribution of DMEs, we present data

256 for male mice at 10 weeks as an example. Fig. 6 displays the distribution of DMEs in liver,

257 intestine and kidney tissue. In liver, CYP2C29 > CYP2D22 > CYP3A11 CYP2E1 CYP1B1 > 
CYP7A1 > CYP27A1 $\approx$ CYP3A25; UGT2B5 > UGT2B1 > UGT1A6a > UGT1A1 > UGT2B34

$259 \approx$ UGT2B36 > UGT2A3 > UGT1A9 $\approx$ UGT1A5 > UGT1A2 (lower limit of quantification,

260 LLOQ); SULT1A1 > SULT1D1 > SULT1B1 (LLOQ). The protein contents of UGT2B5,

261 UGT2B1, UGT1A6a, CYP2C29, CYP2D22, UGT1A1 and SULT1A1 were the highest. In the

262 intestine, CYP1B1 > CYP2D22 > CYP3A11 > CYP3A25 $\approx$ CYP7A1 >

263 CYP2E1/CYP2C29/CYP27A1 (LLOQ); UGT2B34 > UGT1A1 > UGT2A3 > UGT1A2/

264 UGT1A5/ UGT1A6a/UGT1A9/UGT2B1/UGT2B5/UGT2B36 (LLOQ); SULT1B1 > SULT1A1

265 > SULT1D1. The protein contents of CYP1B1, UGT2B34, SULT1B1, CYP2D22, CYP3A11,

266 SULT1A1 and CYP3A25 were the highest. In the kidney, CYP1B1 > CYP2E1 > CYP7A1 >

267 CYP2C29/CYP2D22/CYP3A11/CYP3A25/CYP27A1 (LLOQ). In the kidney, UGT1A2 was

268 detected, but the other UGT isoforms were below the lower limit of quantification; for SULT

269 isoforms, SULT1D1 was detected, but the others were all below the lower limit of quantification.

270 SULT1D1, CYP1B1, CYP2E1, CYP7A1 and UGT1A2 had the highest protein expression levels.

271 DME variations based on sex. Fig. 6 shows that the the sex-related changes in DMEs have a

272 similar trend in both the WT and KRAS mice at 10 weeks of age. Therefore, we mainly discuss

273 the differences in protein content in WT mice. In kidney tissue, CYP2E1 was male-predominant,

274 while UGT1A2 was female-predominant. In liver tissue, the content of UGT2B1 was

275 significantly higher in the male mice than in the female mice.

276 Variations in DME protein content with increased age. In the liver, CYP7A1 increased with

277 increasing age in the male mice (Fig. 7G); UGT1A9 showed a decreasing trend with increasing

278 age (Fig. 7L). In the intestine, the CYP isoforms showed no significant changes at different ages

279 (Fig. 8A-E). UGT2B34 showed a decreasing trend in the male WT and KRAS mice with 
280 increasing age (Fig. 8H), and SULT1A1 showed an increasing trend with increasing age (Fig. 8I).

281 In the kidney, CYP1B1 displayed a decreasing trend in the male WT and KRAS mice with

282 increasing age (Fig. 8L). Supplemental Fig. 6 shows the changes of protein amount with aging in

283 female WT and KRAS mice. In the liver, the protein amount of CYP2C29 decreased 2.9-fold at

28415 weeks compared to that at 5 weeks in the WT mice. This pattern of changes did not appear in

285 the KRAS mice. High individual differences in the protein amounts in female mice were

286 observed. There are no significant differences at different ages. 
288 In this study, we systematically investigated the alterations of DMEs in KRAS mice of

289 different ages and sexes, with the aim of providing a better explanation for the clinically observed

290 variation in the efficacy and toxicity of anticancer drugs in KRAS-mutant lung cancer patients.

291 Currently, limited information is available concerning the changes in DMEs in patients with

292 KRAS mutant lung cancer.

293 The absolute protein contents of 21 metabolic enzymes in KRAS mice were simultaneously

294 determined by the LC-MS/MS approach. In our study, the protein expression levels of CYP2C29

295 and CYP3A11 were significantly downregulated in the male KRAS mice at 26 weeks of age. A

296 previous study indicated that hepatic DMEs are reduced during infection and inflammation in

297 humans, rats, and mice (Nozomu Moriya et al. 2012). Similar results were also reported that a

298 decrease in Cyp gene expression and enzymatic activity was observed in a dextran sulfate sodium

299 (DSS)-induced mouse model of ulcerative colitis (Yoshiiki K. et al. 2014). CYP2C29 is the major

300 arachidonate CYP2C epoxygenase in mice (Komal S. et al. 2009). The decreased expression of

301 CYP2C29 is closely related to the occurrence and development of inflammation. Related studies

302 have shown that this decreased expression may be triggered by an increased production of

303 inflammatory cytokines (Yoshiiki K. et al. 2014). CYP3A11 plays a vital role in the metabolism

304 of various clinical anticancer drugs, such as erlotinib, cisplatin, sorafenib. The drug concentration

305 in serum would change accordingly with enzymatic expression. The declining expression of

306 CYP3A11 in the KRAS mice may cause some differences in efficacy or even side effects. With

307 respect to the SULT family, the protein expression and activities of SULT1A1 and SULT1D1 
308 were upregulated in the male KRAS mice at 20 and 26 weeks. A number of studies have been

309 conducted on SULT in different cancers, but many conflicting outcomes have been reported (Y.

310 Jiang et al. 2010). Some authors showed a potential association between SULT1A1

311 polymorphisms and breast cancer, but inconsistent results also exist (Y. Jiang et al. 2010).

312 Relevant studies have reported that SULT1A3 may be a diagnostic marker for osteosarcoma, and

313 SULT1A3 protein upregulation is closely related to the occurrence and development of cancer

314 (X. Chen et al. 2014; C. Xie et al. 2017). SULT1D1 is a pseudogene in humans, Sult1d1 encodes

315 protein expression in mice, and its functions are similar to those of human SULT1A3 (S. Wong et

316 al. 2010). Our results also revealed increased protein expression of SULT1D1 in male mice after

317 KRAS mutation. This finding is beneficial to explain the metabolic characteristics of SULT1D1-

318 metabolized drugs in KRAS mice. The expression of SULT1A3 should be further explored in

319 KRAS-mutant lung cancer patients.

320 To further explore the changes in enzymatic activity, we used specific probe substrates to

321 detect the enzymatic status in the KRAS mice. In our present study, we found the

322 CYP2C29/CYP3A11/SULT1A1/SULT1D1 displayed significant changes in protein expression in

323 the liver of male WT and KRAS mice. Therefore, we are intended to research their activities in

324 the liver tissue. We failed to find an authoritative and specific probe to study the activity of

325 CYP2C29. Chlorzoxazone and Propofol are usually used as specific substrates to study the

326 activities of CYP2E1 and UGT1A9. Their good correlation between protein expression and

327 activities indicated the protein quantification results are credible. Therefore, we select them for

328 the enzyme activity test. Notably, SULT1A1 and SULT1D1 activity was upregulated at 20 and 26

329 weeks in the male KRAS mice (Fig. 3 and Supplemental Fig. 4). This result was consistent with 
330 their protein expression levels. In this context, a high degree of correlation was observed between

331 the enzymatic activity and protein level (Fig. 4). For the poor correlation between the enzyme

332 activity and protein level of CYP3A11, the nonspecificity of the substrate may be a possible

333 reason. The FDA (USA) reported that testosterone was metabolized by CYP3A4 and CYP3A5. In

334 addition, the protein structure could affect the activity. CYP3A4 showed a significant genetic

335 polymorphism in individuals, causing a flexible three-dimensional structure of CYP3A4 (Werk

336 and Cascorbi 2014). Moreover, the genetic polymorphism of CYP2D6 (ortholog of CYP2D22 in

337 mice) could induce variations in the expression or function of CYP3A4 (Werk and Cascorbi

338 2014). Generally, these findings indicate that the protein expression levels of some DMEs could

339 be applied to forecast the enzymatic activities regarding drug metabolism. Changes in the ability

340 of DMEs to metabolize drugs could lead to differences in the ADME properties of drugs, thereby

341 affecting drug efficacy and toxicity in the body.

342 The expression of DMEs is regulated by the binding of xenobiotics to receptors, such as the

343 aryl hydrocarbon receptor (AHR), the murine pregnane $\mathrm{X}$ receptor (PXR), peroxisome

344 proliferator-activated receptor (PPAR $\alpha$ ) and bile acid receptor (FXR) (S. Anakk et al. 2003; C.

345 HANDSCHIN 2003; HONKAKOSKI and NEGISHI 2000). The decrease in receptor levels may

346 contribute to the emergence of changes in DME expression (S. Anakk et al. 2003; L. Li et al.

347 2009; J. E. Moscovitz et al. 2018). Moreover, some reports have suggested that disease status

348 (e.g., cancer and inflammation) can affect the expression and activity of DMEs via specific

349 receptors (Lamba et al. 2016; H. Chen et al. 2014; A. Schröder et al. 2011). Therefore, we further

350 studied the changes in receptor expression after KRAS mutation. In our study, the protein

351 expression levels of AHR, FXR and PPAR $\alpha$ were downregulated in the livers of the male KRAS 
352 mice compared to the WT mice at 26 weeks. This phenomenon was not significant in the female

353 KRAS mice. Major xenobiotic-sensing transcription factors, such as AHR and PXR, are involved

354 in the regulation of the protein expression of DMEs. Related reports revealed that most core

355 DMEs were positively correlated with AHR, PXR and PPAR $\alpha$, and their protein expression was

356 downregulated in nearly 50\% of the patients with hepatocellular carcinoma (HCC) (H. Chen et al.

357 2014; D. G. Hu et al. 2018; S. Zhong et al. 2016). Activation of AHR could induce the

358 upregulation of Cyp1a/3a/Ugt1a1 mRNA expression, which would therefore not occur in Ahr-

359 null mice (C.D. Klaassen and A.L. Slitt 2005; Nakajima et al. 2003). PPAR $\alpha$ and PXR were

360 implicated in the regulation of CYP3A/4A/1A1/2B6/2C8/2C9/2C19/UGT1A1 induction (J. E.

361 Moscovitz et al. 2018). FXR, an important regulator of lipid and glucose homeostasis, is involved

362 in the expression of CYP7A1 and CYP27A1 (Sánchez 2018). Hence, in our study, we speculate

363 that these variations in DME expression may be regulated by decreased receptors of AHR, FXR

364 and PPAR $\alpha$.

365 For sex-difference, CYP2E1 showed significant male-specificity in kidney tissue. CYP2E1

366 mediates the metabolism of many low molecular weight organic compounds (such as ethanol and

367 acetone) and some drugs (such as p-nitrophenol, caffeine, chlorzoxazone, etc.) (S. Löfgren et al.

368 2004; Zuber et al. 2002). Therefore, in regard to the intake of these exogenous substances, we

369 should consider the effects related to sex differences in patients. For UGT enzymes, UGT2B1

370 exhibited male-predominant expression in the liver tissue. Conversely, UGT1A1 and UGT1A5

371 expression in the liver and UGT1A2 in the kidney are female-predominant, whereas UGT2B1

372 exhibited male-predominant expression in liver tissue. These results are consistent with previous

373 reports (D. B. Buckley and C. D. Klaassen 2007, 2009). The female-predominant UGT1A1 
374 expression accounts for the higher bilirubin-UGT activity in females. UGT1A and UGT2B are

375 the primary families of UGT enzymes, involved in the inactivation of $>30 \%$ of drugs currently

376 used in the clinic (C. Guillemette et al. 2014). These sex-specific expressions may be crucial in

377 understanding the mechanisms by which many drugs display variations in metabolism and

378 clearance.

379 For age-related difference, except for UGT1A9, the majority of DMEs showed no significant 380 changes from 5 to 26 weeks of age in female and male WT and KRAS mice. UGT1A9, major

381 UGT isoforms expressed in the liver ( $\sim \%$ of hepatic UGT expression), is responsible for the 382 glucuronidation of multiple endogenous substances (e.g., thyroid hormones) and drugs (e.g., 383 acetaminophen and propofol) (S. Cho et al. 2016). The activity and protein expression of 384 UGT1A9 appeared to decrease in the liver of female and male WT mice. Related studies 385 indicated that UGT1A9 activity showed a downward trend from 6 weeks to 52 weeks in mice 386 with a FVB background (H. Zheng et al. 2018). Therefore, the appropriate dosage should be 387 considered when patients of different ages are prescribed drugs metabolized by UGT1A9.

388 For tissue-related differences, abundant CYP enzymes were expressed in the liver, 389 predominantly CYP2D22, CYP2C29, CYP2E1 and CYP3A11 (Fig. 6A-B). A previous studies 390 also demonstrated that the protein contents of these isoforms were high in the liver (J. Chen et al. 391 2017; C. Gröer et al. 2014). In the intestine, the CYP1B1, CYP2D22, CYP2E1 and CYP3A11 392 protein levels were significantly higher than the levels of other proteins. Mouse phase I enzymes 393 (CYP2D22, CYP2E1 and CYP3A11) are orthologs of the corresponding human enzymes 394 (CYP2D6, CYP2E1 and CYP3A4), in charge of major phase I-dependent metabolism in 395 marketed drugs (Liu 2013; G. Ruaño et al. 2012). Hence, optimal drug administration routes 
396 should be considered when these enzymes are involved in the inactivation or activation of drugs.

397

398 CONCLUSION

399 Taken together, our data showed significant decrease in CYP3A11 and CYP2C29, but an 400 increase in SULT1A1 and SULT1D1 in the KRAS mice at 26 weeks. These DMEs all participate 401 in the metabolism of drugs. Therefore, we hope that these results could provide useful guidance 402 or a theoretical basis for further drug research and implementation. 


\section{ACKNOWLEDGMENTS}

404 We thank Tongmeng Yan of State Key Laboratory of Quality Research in Chinese Medicine,

405 Macau University of Science and Technology, Macau (SAR), China for the method of S9 sample 406 preparation.

407 We thank Guoxin Huang of State Key Laboratory of Quality Research in Chinese Medicine, 408 Macau University of Science and Technology, Macau (SAR), China for checking English 409 language problems. 


\section{REFERENCES}

411

412

413

414

415

416

417

418

419

420

421

422

423

424

425

426

427

428

429

430

431

432

433

434

435

436

437

438

439

440

441

Adrian Schröder, Johannes Wollnik, Clemens Wrzodek, Andreas Dräger, Michael Bonin, Oliver Burk, Maria Thomas, Wolfgang E. Thasler, Ulrich M. Zanger and Andreas Zell. 2011. "Inferring Statin-Induced Gene Regulatory Relationships in Primary Human Hepatocytes.” Bioinformatics. 27(18): 2473-7.

April D. Lake, Petr Novak, Craig D. Fisher, Jonathan P. Jackson, Rhiannon N. Hardwick, D. Dean Billheimer, Walter T. Klimecki, and Nathan J. Cherrington. 2011. "Analysis of Global and Absorption, Distribution , Metabolism , and Elimination Gene Expression in the Progressive Stages of Human Nonalcoholic Fatty Liver Disease.” Drug Metab Dispos. 39(10): 1954-60.

A Tourancheau, G Margaillan, M Rouleau, I Gilbert, L Villeneuve, E Lévesque, A Droit and C Guillemette. 2016. "Unravelling the Transcriptomic Landscape of the Major Phase II UDP-Glucuronosyltransferase Drug Metabolizing Pathway Using Targeted RNA Sequencing.” Pharmacogenomics Journal. 16(1): 60-70.

Beatrice A. Nyagode, Roya Jahangardi, Matthew D. Merrell, Malu G. Tansey \& Edward T. Morgan. 2014. "Selective Effects of a Therapeutic Protein Targeting Tumor Necrosis Factor-Alpha on Cytochrome P450 Regulation during Infectious Colitis: Implications for Disease-Dependent Drug-Drug Interactions.” Pharmacology Research and Perspectives. 2(1): 1-12.

By Hassan R. Dhaini, Dafydd G. Thomas, Thomas J. Giordano, Timothy D. Johnson, J. Sybil Biermann, Kirsten Leu, Paul F. Hollenberg, and Laurence H. Baker. 2003. "Cytochrome P450 CYP3A4/5 Expression as a Biomarker of Outcome in Osteosarcoma." Journal of Clinical Oncology. 21(13): 2481-85.

C.D. Klaassen and A.L. Slitt. 2005. "Regulation of Hepatic Transporters by Xenobiotic Receptors.” Curr Drug Metab. 6(4): 309-28.

C. Gröer, D. Busch, M. Patrzyk, K. Beyer, A. Busemann, C.D. Heidecke, M. Drozdzik, W. Siegmund, S. Oswald. 2014. “Absolute Protein Quantification of Clinically Relevant Cytochrome P450 Enzymes and UDP-Glucuronosyltransferases by Mass SpectrometryBased Targeted Proteomics.” Journal of Pharmaceutical and Biomedical Analysis. 100: 393-401.

C Guillemette, É Lévesque and M Rouleau. 2014. "Pharmacogenomics of Human Uridine Diphospho-Glucuronosyltransferases and Clinical Implications.” Clinical Pharmacology

Peer) reviewing PDF | (2020:01:45080:3:0:NEW 18 Sep 2020) 
and Therapeutics. 96(3): 324-39.

443

444

445

446

447

448

449

450

451

452

453

454

455

456

457

458

459

460

461

462

CHRISTOPH HANDSCHIN AND URS A. MEYER. 2003. "Induction of Drug Metabolism: the Role for Nuclear Receptors.” Pharmacol Rev. 55(4): 649-73.

Cong Xie, Tong-meng Yan, Jia-mei Chen, Xiao-yan Li, Juan Zou, Li-jun Zhu, Lin-lin Lu, Ying Wang, Fu-yuan Zhou, Zhong-qiu Liu \& Ming Hu. 2017. "LC-MS/MS Quantification of Sulfotransferases Is Better than Conventional Immunogenic Methods in Determining Human Liver SULT Activities: Implication in Precision Medicine.” Scientific Reports. 7(1): 1-14.

Court, Michael H. 2010. "Interindividual Variability in Hepatic Drug Glucuronidation: Studies into the Role of Age, Sex, Enzyme Inducers, and Genetic Polymorphism Using the Human Liver Bank as a Model System.” Drug Metabolism Reviews. 42(1): 209-224.

David B. Buckley and Curtis D. Klaassen. 2007. "Tissue- and Gender-Specific MRNA Expression of UDP-Glucuronosyltransferases (UGTs) in Mice.” Drug Metabolism and Disposition. 35(1): 121-27.

David B. Buckley and Curtis D. Klaassen. 2009. "Mechanism of Gender-Divergent UDPGlucuronosyltransferase MRNA Expression in Mouse Liver and Kidney.” Drug Metabolism and Disposition. 37(4): 834-40.

Dong Gui Hu , Shashikanth Marri , Ross A. McKinnon , Peter I. Mackenzie , and Robyn Meech. 2018. "Deregulation of the Genes That Are Involved in Drug Absorption, Distribution, Metabolism, and Excretion (ADME Genes) in Hepatocellular Carcinoma." Journal of Pharmacology and Experimental Therapeutics. 368(3): 363-381.

Gualberto Ruaño, David Villagra, Bonnie Szarek, Andreas Windemuth, Mohan Kocherla, Krystyna Gorowski, Christopher Berrezueta, Harold I Schwartz, and John Goethe. 2012. "Physiogenomic Analysis of CYP450 Drug Metabolism Correlates Dyslipidemia with Pharmacogenetic Functional Status in Psychiatric Patients.” Biomark Medicine. 5(4): 43949.

Haihui Zheng, Liping Wang, Sijing Zeng, Jiamei Chen, Haojia Wang, Jia Yu, Xia Gong, Huangyu Jiang, Xia Yang, Xiaoxiao Qi, Ying Wang, Linlin Lu, Ming Hu, Lijun Zhu, and Zhongqiu Liu. 2018. "Age-Related Changes in Hepatic Expression and Activity of Drug Metabolizing Enzymes in Male Wild-Type and Breast Cancer Resistance Protein Knockout Mice.” Biopharmaceutics and Drug Disposition. 39(7): 344-53.

Hanna K. Sanoff, Janine M. Davies, Christine Walko, William Irvin, Larry Buie, Kimberly Keller, Anastasia Ivanova, Wing-Keung Chiu, Bert H. O’Neil, Thomas E. Stinchcombe, 
475

476

477

478

479

480

481

482

483

484

485

486

487

488

489

490

491

492

493

494

495

496

497

498

499

500

501

502

503

504

505

506

E. Claire Dees. 2010. "A Phase I Evaluation of the Combination of Vinflunine and Erlotinib in Patients with Refractory Solid Tumors.” Investigational New Drugs. 29(5): 978-83.

Hong Chen, Zhong-Yang Shen, Wang Xu, Tie-Yan Fan, Jun Li, Yuan-Fu Lu, Ming-Liang Cheng, Jie Liu. 2014. "Expression of P450 and Nuclear Receptors in Normal and EndStage Chinese Livers.” World Journal of Gastroenterology. 20(26): 8681-8690.

HONKAKOSKI, Paavo, and Masahiko NEGISHI. 2000. "Regulation of Cytochrome P450 (CYP) Genes by Nuclear Receptors.” Biochemical Journal. 347(2): 321-337.

Hui Li, John D. Clarke, Anika L. Dzierlenga, John Bear, Michael J. Goedken, Nathan J. Cherrington. 2017. "In Vivo Cytochrome P450 Activity Alterations in Diabetic Nonalcoholic Steatohepatitis Mice.” Journal of Biochemical and Molecular Toxicology. 31(2): 1-9.

Jamie E. Moscovitz, Amit S. Kalgutkar, Kelly Nulick, Nathaniel Johnson, Zhiwu Lin, Theunis C. Goosen and Yan Weng. 2018. "Establishing Transcriptional Signatures to Differentiate PXR- , CAR- and AhR-Mediated Regulation of Drug Metabolism and Transport Genes in Cryopreserved Human Hepatocytes.” Pharmacol Exp Ther. 365(2): 262271.

Jean-Louis Pujol, Patrick Viens, Paul Rebattu, Scott A. Laurie, Ronald Feld, Anne Deneulin and Abderrahim Fandi. 2006. "Gefitinib (IRESSA) with vinorelbine or vinorelbine/cisplatin for chemotherapy-naive non-small cell lung cancer patients.” Thorac Oncol. 1(5): 417-24.

Jiamei Chen, Lijun Zhu, Xiaoyan Li, Haihui Zheng, Tongmeng Yan, Cong Xie, Sijing Zeng, Jia Yu, Huangyu Jiang, Linlin Lu, Xiaoxiao Qi, Ying Wang, Ming Hu, Zhongqiu Liu. 2017. "High-Throughput and Reliable Isotope Label-Free Approach for Profiling 24 Metabolic Enzymes in FVB Mice and Sex Differences.” Drug Metabolism and Disposition. 45(6): 624-34.

Kennedy, M J. 2008. "Hormonal Regulation of Hepatic Drug-Metabolizing Enzyme Activity During Adolescence.” Clin Pharmacol Ther. 84(6): 662-73.

Komal Sodhi, Kazuyoshi Inoue, Katherine H. Gotlinger, Martina Canestraro, Luca Vanella, Dong Hyun Kim, Vijay L. Manthati, Sreenivasulu Reddy Koduru, John R. Falck, Michal L. Schwartzman, and Nader G. Abraham. 2009. "Epoxyeicosatrienoic Acid Agonist Rescues the Metabolic Syndrome Phenotype of HO-2-Null Mice.” J Pharmacol Exp Ther 331(3): 906-16. 
508

509

510

511

512

513

514

515

516

517

518

519

520

521

522

523

524

525

526

527

528

529

530

531

532

533

534

535

536

537

538

539

540

541

542

543

544

Manickam Aravagiri, Margaret A. Kirshner, BA, Lon S. Schneider, and Robert R. Bies. 2008. "Sex, race, and smoking impact olanzapine exposure." JOURNAL OF CLINICAL PHARMACOLOGY. 48(2): 157-65.

Lamba, V., B. Jia, and F. Liang. 2016. "STAT5A and STAT5B Have Opposite Correlations with Drug Response Gene Expression.” Biochemical and Biophysical Research Communications. 479(2):117-124.

Lan Tang, Qian Feng, Jie Zhao, Lingna Dong, Wei Liu, Caihua Yang, Zhongqiu Liu. 2012. "Involvement of UDP-Glucuronosyltranferases and Sulfotransferases in the Liver and Intestinal First-Pass Metabolism of Seven Flavones in C57 Mice and Humans in Vitro.” Food and Chemical Toxicology. 50(5): 1460-67.

Leisa Johnson, Kim Mercer, Doron Greenbaum, Roderick T. Bronsonk, Denise Crowley, David A. Tuveson \& Tyler Jacks. 2001. "Somatic Activation of the K-Ras Oncogene Causes Early Onset Lung Cancer in Mice.” Nature. 410(6832): 1111-16.

Li Ding, Gad Getz, David A. Wheeler, Elaine R. Mardis, Michael D. McLellan, Kristian Cibulskis, Carrie Sougnez, Heidi Greulich, Donna M. Muzny, Margaret B. Morgan, Lucinda Fulton, Robert S. Fulton, Qunyuan Zhang, Michael C. Wendl, Michael S. Lawrence, David E. Larson, Ken Chen, David J. Dooling, Aniko Sabo, Alicia C. Hawes, Hua Shen, Shalini N. Jhangiani, Lora R. Lewis, Otis Hall, Yiming Zhu, Tittu Mathew, Yanru Ren, Jiqiang Yao, Steven E. Scherer, Kerstin Clerc, Ginger A. Metcalf, Brian Ng, Aleksandar Milosavljevic, Manuel L. Gonzalez-Garay, John R. Osborne, Rick Meyer, Xiaoqi Shi, Yuzhu Tang, Daniel C. Koboldt, Ling Lin, Rachel Abbott, Tracie L. Miner, Craig Pohl, Ginger Fewell, Carrie Haipek, Heather Schmidt, Brian H. DunfordShore, Aldi Kraja, Seth D. Crosby, Christopher S. Sawyer, Tammi Vickery, Sacha Sander, Jody Robinson, Wendy Winckler, Jennifer Baldwin, Lucian R. Chirieac, Amit Dutt, Tim Fennell, Megan Hanna, Bruce E. Johnson, Robert C. Onofrio, Roman K. Thomas, Giovanni Tonon, Barbara A. Weir, Xiaojun Zhao, Liuda Ziaugra, Michael C. Zody, Thomas Giordano, Mark B. Orringer, Jack A. Roth, Margaret R. Spitz, Ignacio I. Wistuba, Bradley Ozenberger, Peter J. Good, Andrew C. Chang, David G. Beer, Mark A. Watson, Marc Ladanyi, Stephen Broderick, Akihiko Yoshizawa, William D. Travis, William Pao, Michael A. Province, George M. Weinstock, Harold E.Varmus, Stacey B.Gabrie, Eric S. Lander, RichardA.Gibbs,MatthewMeyerson, Richard K.Wilson. 2008. "Somatic Mutations Affect Key Pathways in Lung Adenocarcinoma.”Nature. 455(7216): 1069-1075.

Linhao Li, Joseph D. Stanton, Antonia H. Tolson, Yuan Luo and Hongbing Wang. 2009. "Bioactive Terpenoids and Flavonoids from Ginkgo Biloba Extract Induce the Expression of Hepatic Drug-Metabolizing Enzymes through Pregnane X Receptor, Constitutive Androstane Receptor, and Aryl Hydrocarbon Receptor-Mediated Pathways.” 
Pharmaceutical Research. 26(4): 872-82.

Liu Jin, Gregory J. Tawa, and Anders Wallqvist. 2013. "Identifying Cytochrome P450 Functional Networks and Their Allosteric Regulatory Elements.” PLoS ONE. 8(12): 1-11.

Mccormick, Frank. 2015. “KRAS as a Therapeutic Target.” Clin Cancer Res. 21(8): 17971801.

Melanie M Hagleitner, Marieke JH Coenen, Hans J Gelderblom, Remco R Makkinje, Hanneke I Vos, Eveline SJM de Bont, Winnette TA van der Graaf, HW Bart Schreuder, Uta Flucke, Frank N van Leeuwen, Peter M Hoogerbrugge, Henk-Jan Guchelaar, D Maroeska WM te Loo. 2015. "A First Step toward Personalized Medicine in Osteosarcoma: Pharmacogenetics as Predictive Marker of Outcome after ChemotherapyBased Treatment.” Clinical Cancer Research. 21(15): 3436-41.

Min Kyoung Kim, Jeong Yee, Yoon Sook Cho, Hong Won Jang, Ji Min Han and Hye Sun Gwak. 2018. "Risk Factors for Erlotinib-Induced Hepatotoxicit 
Gastroenterology and Hepatology. 15(4): 205.

Sayeepriyadarshini Anakk, Auinash Kalsotra, Qi Shen, Mary T. Vu, Jeffrey L. Staudinger, Peter J. A. Davies, and Henry W. Strobel. 2003. "Genomic Characterization and Regulation of CYP3a13: Role of Xenobiotics and Nuclear Receptors.” The FASEB journal 
612 Wang, Shuo, Xiaoxue Lai, Yihui Deng, and Yanzhi Song. 2020. "Correlation between Mouse

613 Age and Human Age in Anti-Tumor Research: Significance and Method Establishment." $614 \quad$ Life Sciences. 242:117242.

Wanqing Liu, Jacqueline Ramı́rez, Eric R. Gamazon, Snezana Mirkov, Peixian Chen, Kehua Wu, Chang Sun, Nancy J. Cox, Edwin Cook Jr, Soma Das and Mark J. Ratain. 2014. "Genetic Factors Affecting Gene Transcription and Catalytic Activity of UDP-

Waxman, D. J., and M. G. Holloway. 2009. "Sex Differences in the Expression of Hepatic Glucuronosyltransferases in Human Liver.” Human molecular genetics. 23(20): 5558-69.

Werk, A N, and I Cascorbi. 2014. "Functional Gene Variants of CYP3A4.” Clin Pharmacol Ther. 96(3): 340-8.

Wu, Kuo-chen, and Chun-jung Lin. 2018. "ScienceDirect The Regulation of DrugMetabolizing Enzymes and Membrane Transporters by Inflammation: Evidences in Inflammatory Diseases and Age-Related Disorders.” Journal of Food and Drug Analysis. 27(1): 48-59.

Yang, Lun, and Yan Li. 2012. "Sex Differences in the Expression of Drug-Metabolizing and Transporter Genes in Human Liver.” Journal of Drug Metabolism \& Toxicology. 3(3): 1-20. 2010. "Association of Sulfotransferase SULT1A1 with Breast Cancer Risk: A Meta-Analysis Wataru Ochiai, Yoshiaki Machida, Kiyoshi Sugiyama. 2014. "Hepatic Early Inflammation Induces Downregulation of Hepatic Cytochrome P450 Expression and Journal of Pharmaceutical Sciences. 54(1): 17-27. 
643 Zhu, Wei, Haiyan Xu, Stephen W. J. Wang, and Ming Hu. 2010. "Breast Cancer Resistance 644 Protein (BCRP) and Sulfotransferases Contribute Significantly to the Disposition of 645 Genistein in Mouse Intestine.” The AAPS Journal. 12(4): 525-36.

646 Zuber, Roman, Eva Anzenbacherová, and P. Anzenbacher. 2002. "Cytochromes P450 and 647 Experimental Models of Drug Metabolism.” Journal of Cellular and Molecular Medicine. $648 \quad$ 6(2): $189-98$.

649 


\section{Figure 1}

Morphology and H\&E staining of lung tissues from the WT and KRAS mice.

(A-B) Pulmonary morphology in the WT and KRAS mice under a stereoscopic mirror. The arrows pointed out the lung nodules in KRAS mice. $(\mathrm{C}-\mathrm{H})$ Histological and pathological features of the WT and KRAS mice were detected by H\&E staining at 26 weeks. The arrows partly pointed out the hyperproliferative lung cells in KRAS mice.
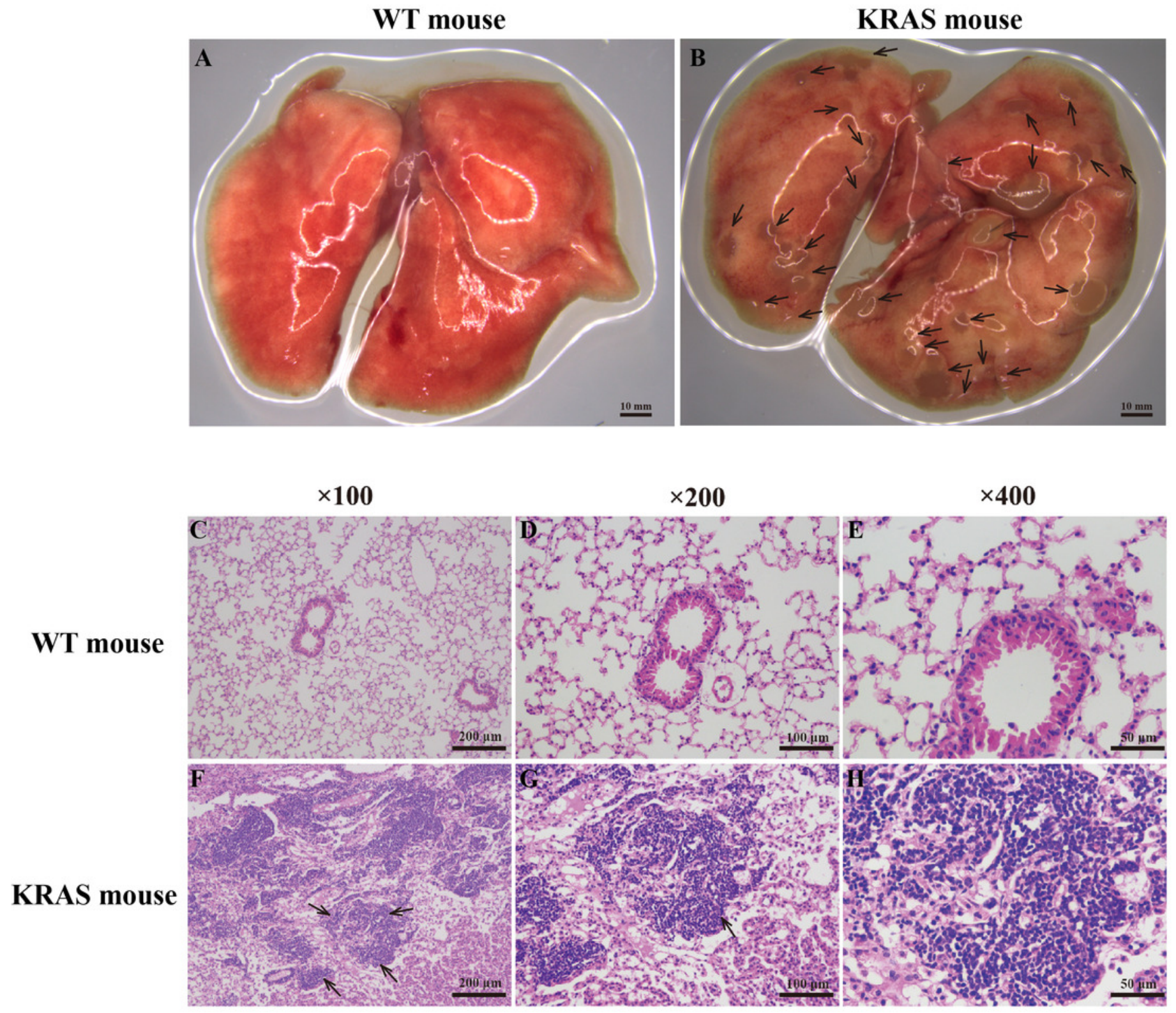


\section{Figure 2}

Alterations in DME protein content with KRAS mutation in the different tissues at different ages.

(A-E) Comparative data analysis of DMEs protein content in the liver of the male WT and KRAS mice was performed using PLS-DA plot at different ages. The solid black dots represent the KRAS mice, and the open black dots represent the WT mice $(n=5)$. (F-H) The relative expression levels of DMEs in the liver, intestine and kidney tissue in the male mice at different ages. Protein levels in the male WT mice $(n=5)$ were normalized to those in the male KRAS mice $(n=5)$. The data were analyzed by independent sample $t$ tests (for normally distributed data) and Mann-Whitney $\mathrm{U}$ analysis (for non-normally distributed data). The symbol "*" indicates a displayed significant difference between the male WT and KRAS mice at the same age, $p<0.05$. 

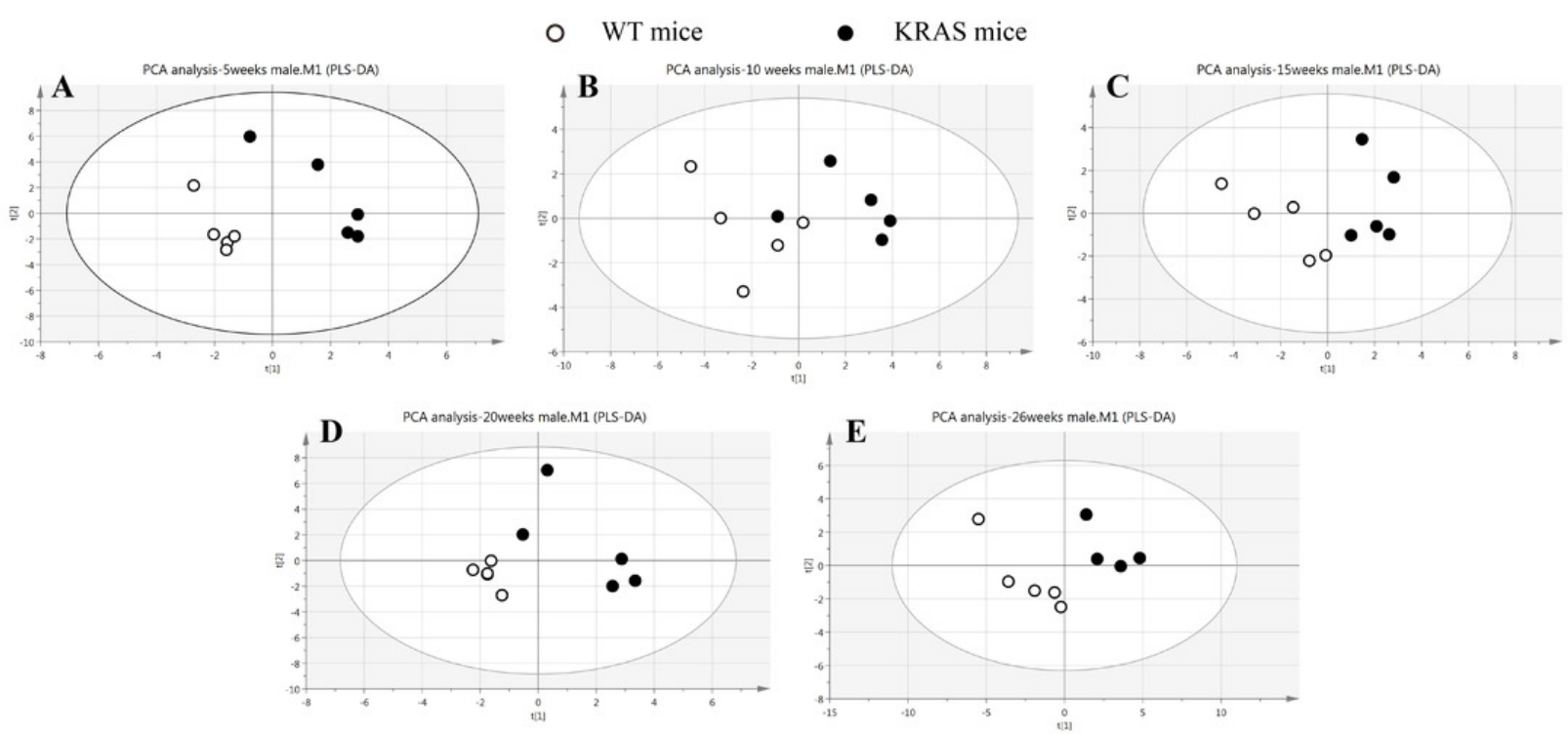

Male mice

$\square$ 5week $\square$ 10week $\square$ 15week $\quad \square$ 20week $\square$ 26week

Kidney
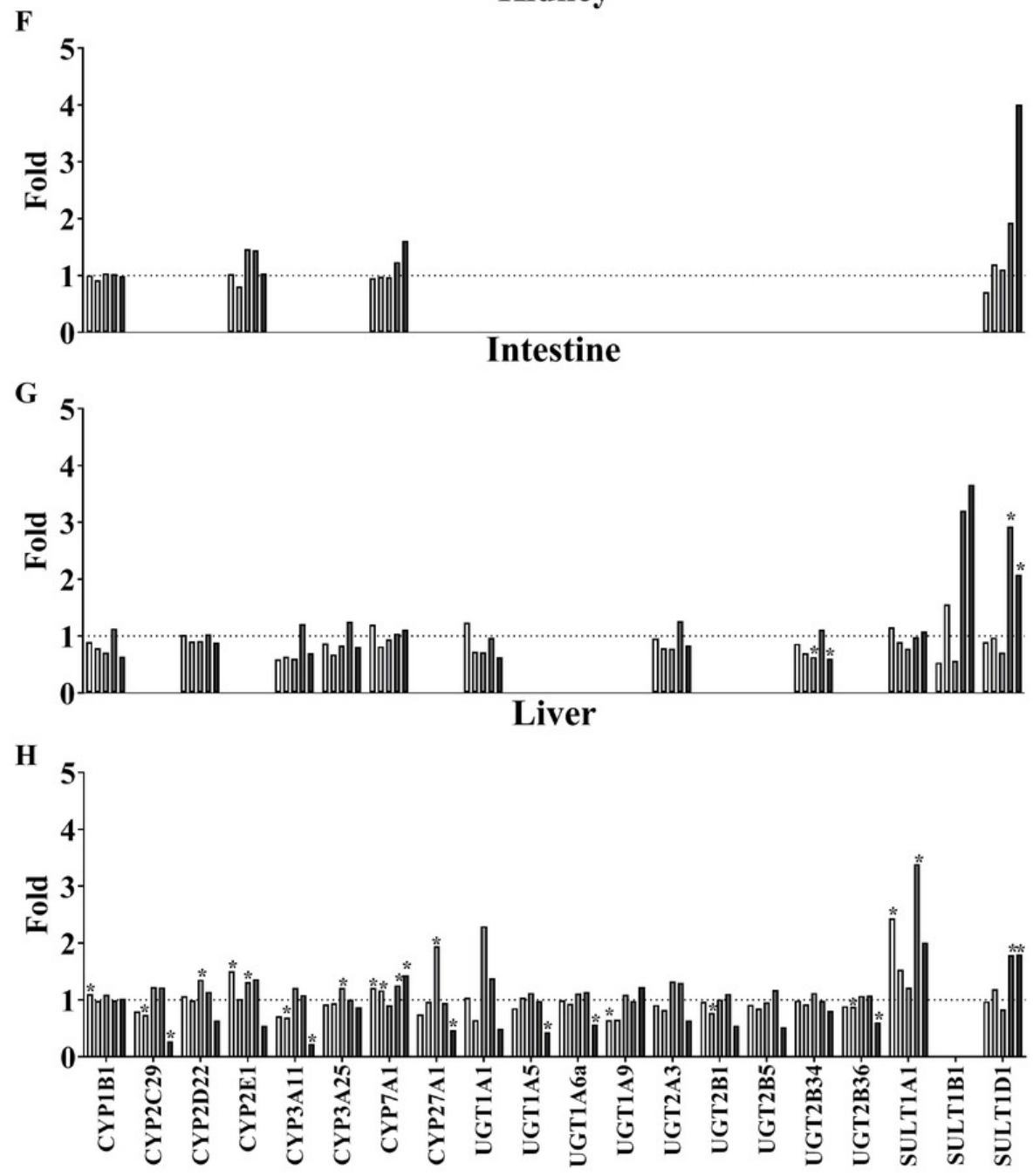


\section{Figure 3}

Changes in the enzyme activity of CYP2E1, CYP3A11, UGT1A9, SULT1A1 and SULT1D1 in liver tissues of the male KRAS and WT mice at different ages $(n=5)$.

The solid line represents male KRAS mice, and the dashed line represents male WT mice. Each data point is presented as the mean \pm SD. For the comparison between KRAS and WT at the same age, the data were analyzed by independent sample $t$ tests (for normally distributed data) and Mann-Whitney $U$ analysis (for non-normally distributed data). The symbol "*" indicates a significant difference between the male WT and KRAS mice at the same age, $p<0.05$. For different ages compared to 5 weeks, the data were analyzed by oneway ANOVA (for normally distributed data) and Kruskal-Wallis $\mathrm{H}$ analysis (for non-normally distributed data). We adjusted the significance level $\alpha$ to 0.0125 according to the Bonferroni correction $(0.05 / 4=0.0125)$. The symbols " $A$ " and "a" indicate significant differences in the male WT and KRAS mice at 10, 15, 20 and 26 weeks relative to 5 weeks, $p<0.0125$. 
- - Male WT

A CYP2E1

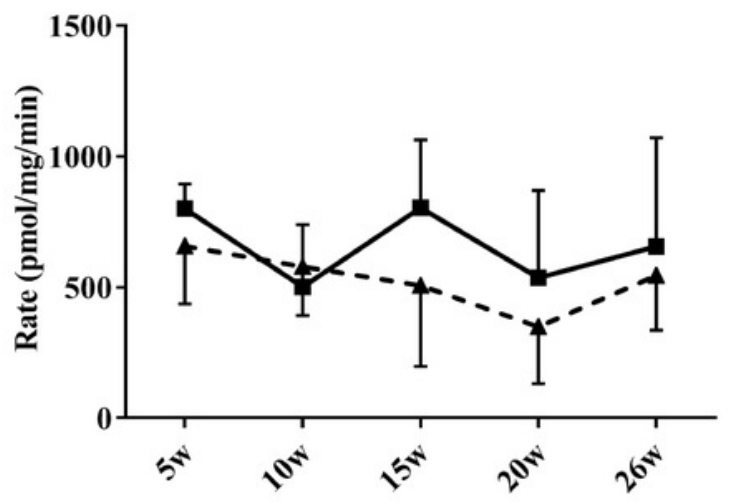

C UGT1A9

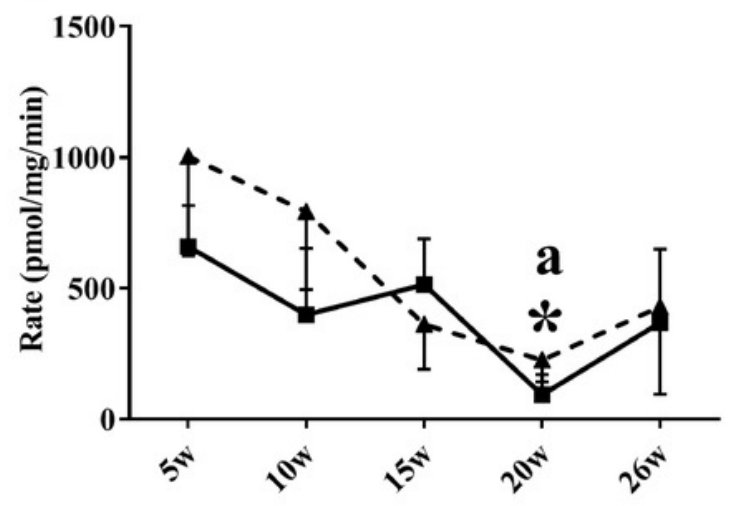

SULT1D1

E

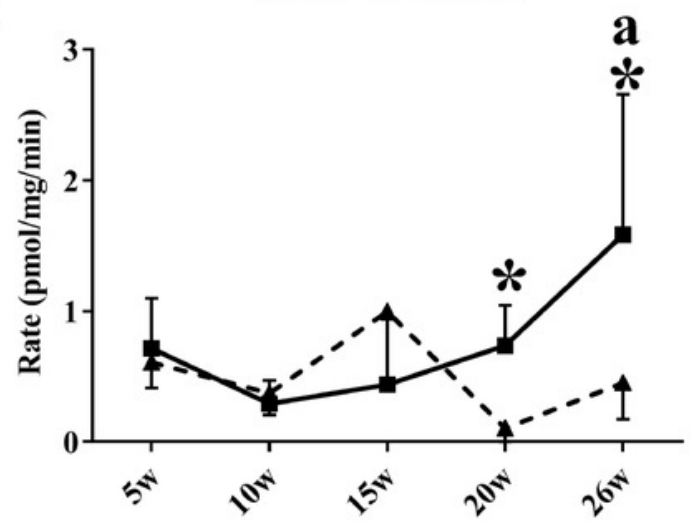

- Male KRAS

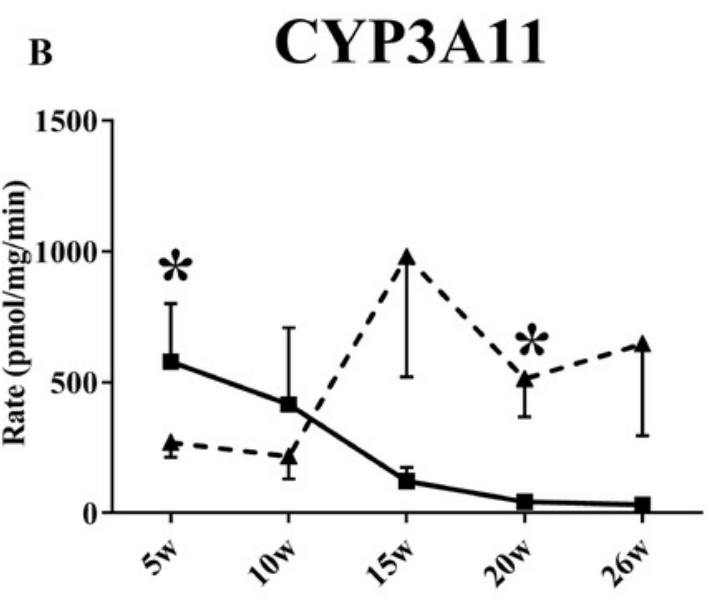

SULT1A1

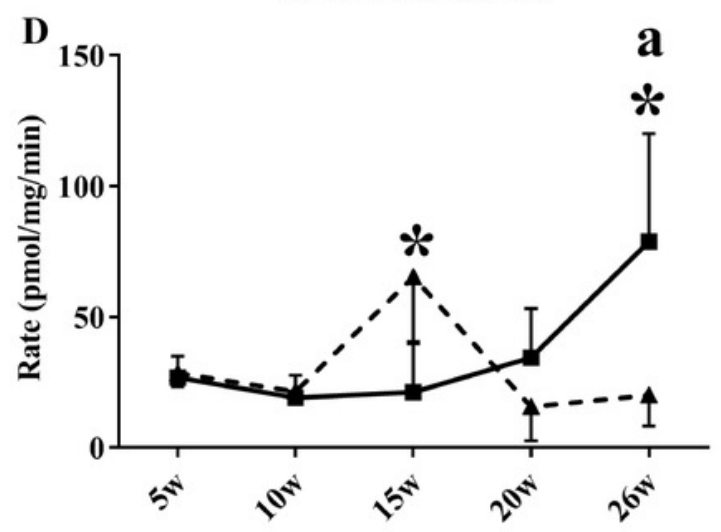




\section{Figure 4}

Correlation between the protein expression and activity of CYP2E1, CYP3A11, UGT1A9, SULT1A1 and SULT1D1 in the liver tissue $(n=100)$.

The correlation between the protein expression and activity included 5, 10, 15, 20 and 26 weeks, which were analyzed together. DME in the liver was determined using an isotope label-free LC-MS/MS method. The enzyme activities of DMEs were measured using probe substrates. All measurements were performed in triplicate and the data are presented as the mean \pm SD. Pearson product correlation and Spearman's rank correlation were used to analyze the correlation. Regression line is shown for significant correlation at $p<0.05$. 

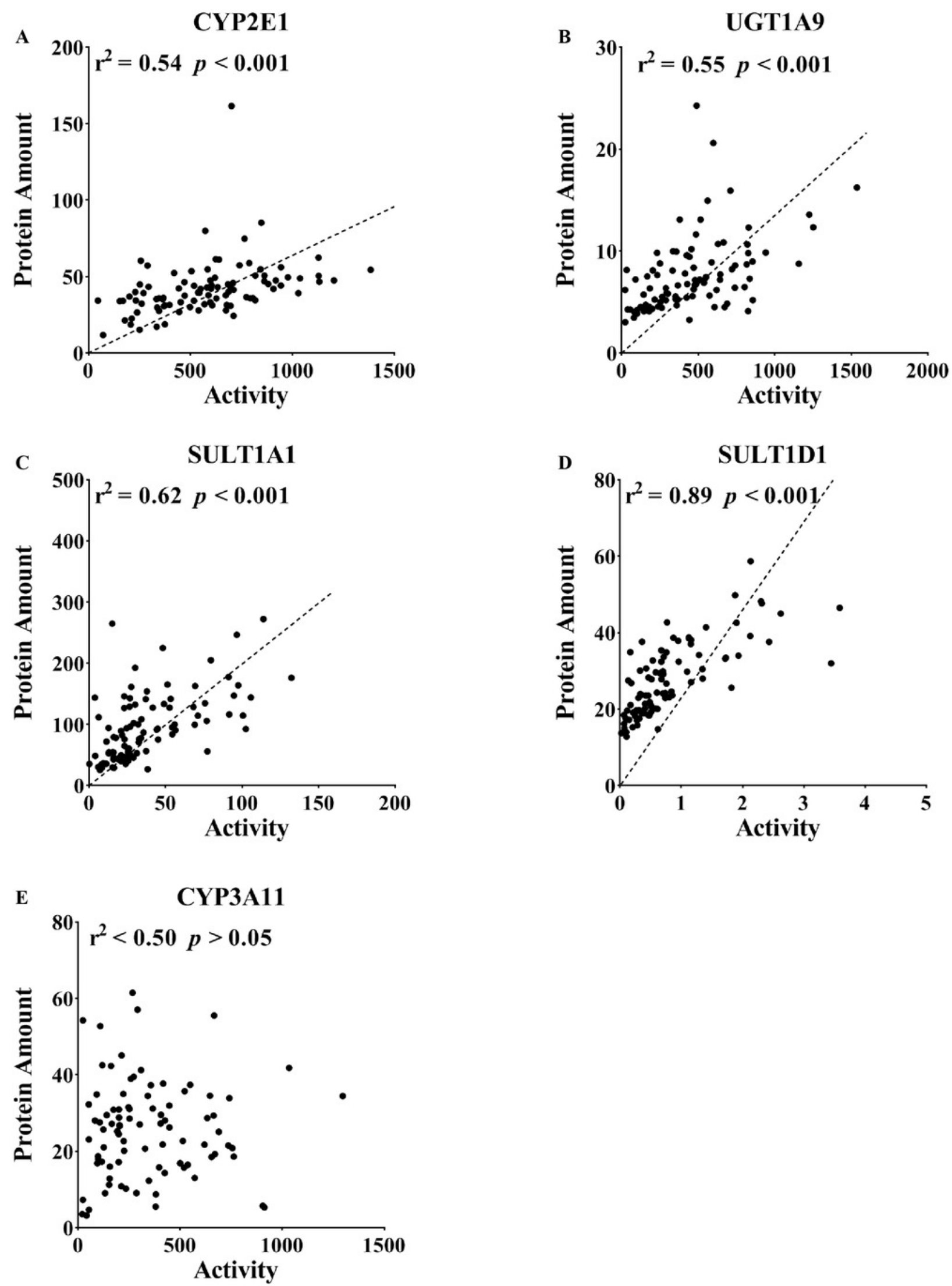


\section{Figure 5}

Protein expression levels of AHR, FXR, PPAR $\alpha$ and PXR in the male WT $(n=5)$ and KRAS mice $(n=4)$ at 26 weeks.

(A) The mprint of five proteins was represented and $\beta$-ACTIN was used as an internal control. (B) The data on protein expression levels was shown as a box chart. The data were analyzed by independent sample $t$ tests (for normally distributed data) and Mann-Whitney $U$ analysis (for non-normally distributed data). The symbol "*" indicates a significance difference of protein expression level in the KRAS mice relative to that in the WT mice, $p<0.05$. 


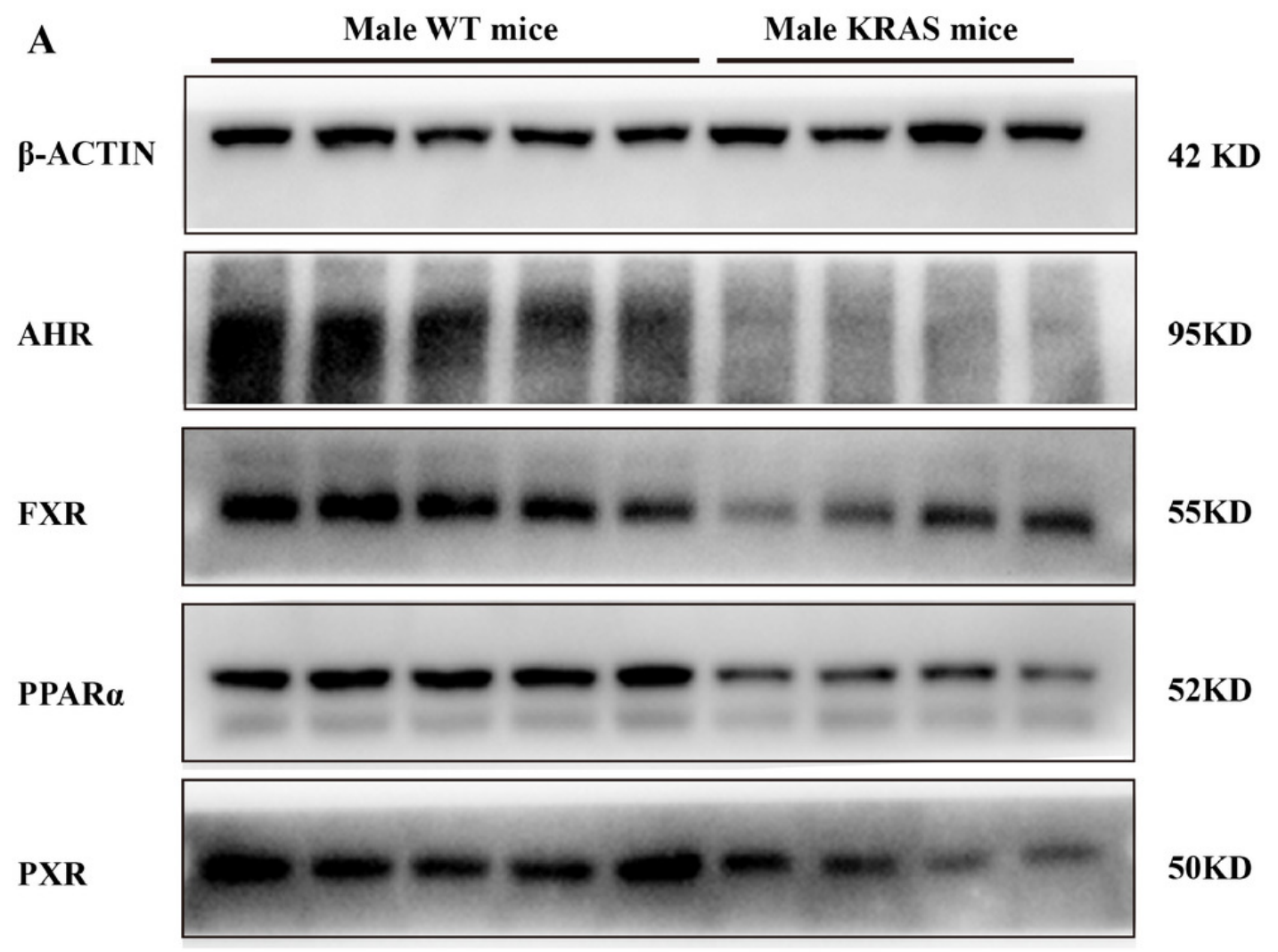

B

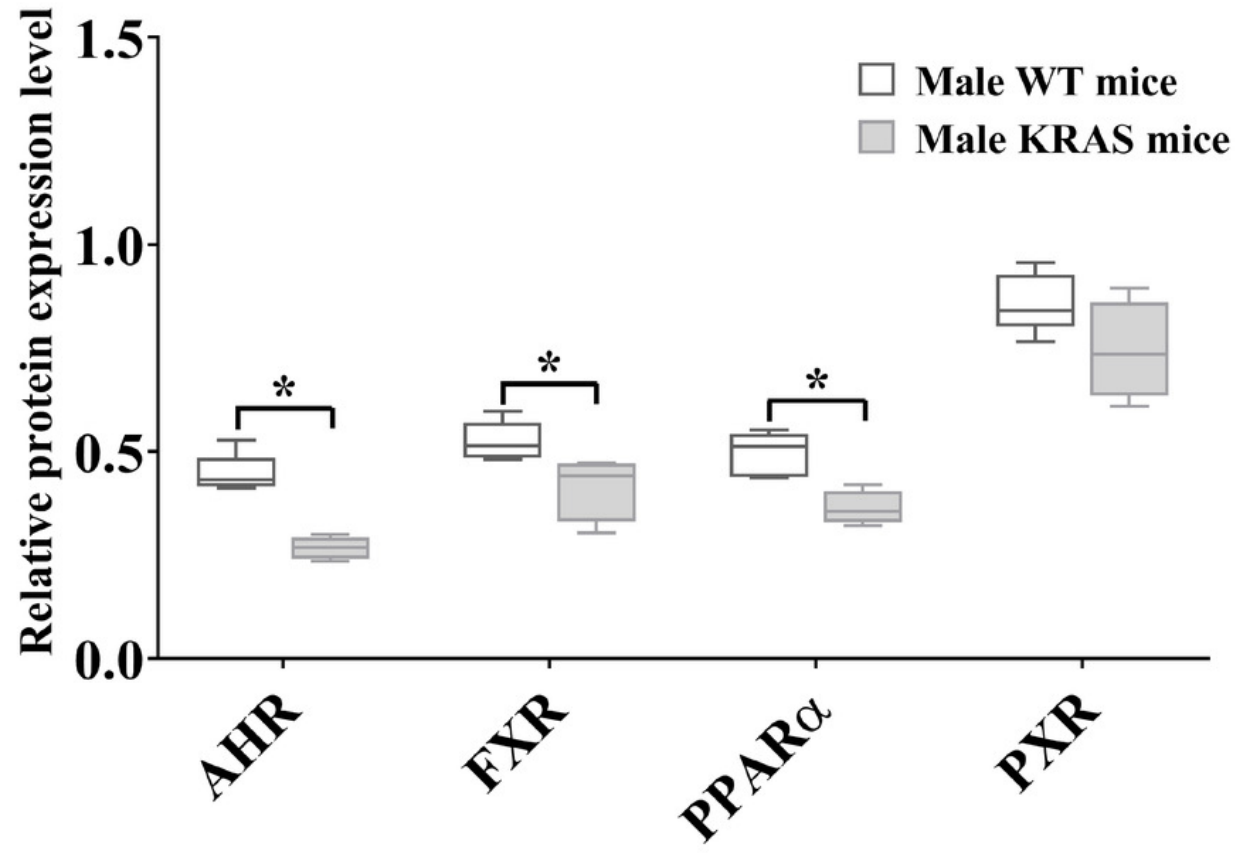




\section{Figure 6}

Protein content of DMEs in different tissues from the KRAS and WT mice with different sexes at 10 weeks, $n=5$.

All measurements were performed in triplicate and the data are presented as the mean \pm SD. (A-B) Protein levels of 8 CYP isoforms in different tissues from the KRAS and WT mice with different sexes. (C-D) Protein levels of 10 UGT isoforms in different tissues from the KRAS and WT mice with different sexes. (E-F) Protein levels of 3 SULT isoforms in different tissues from the KRAS and WT mice with different sexes. 

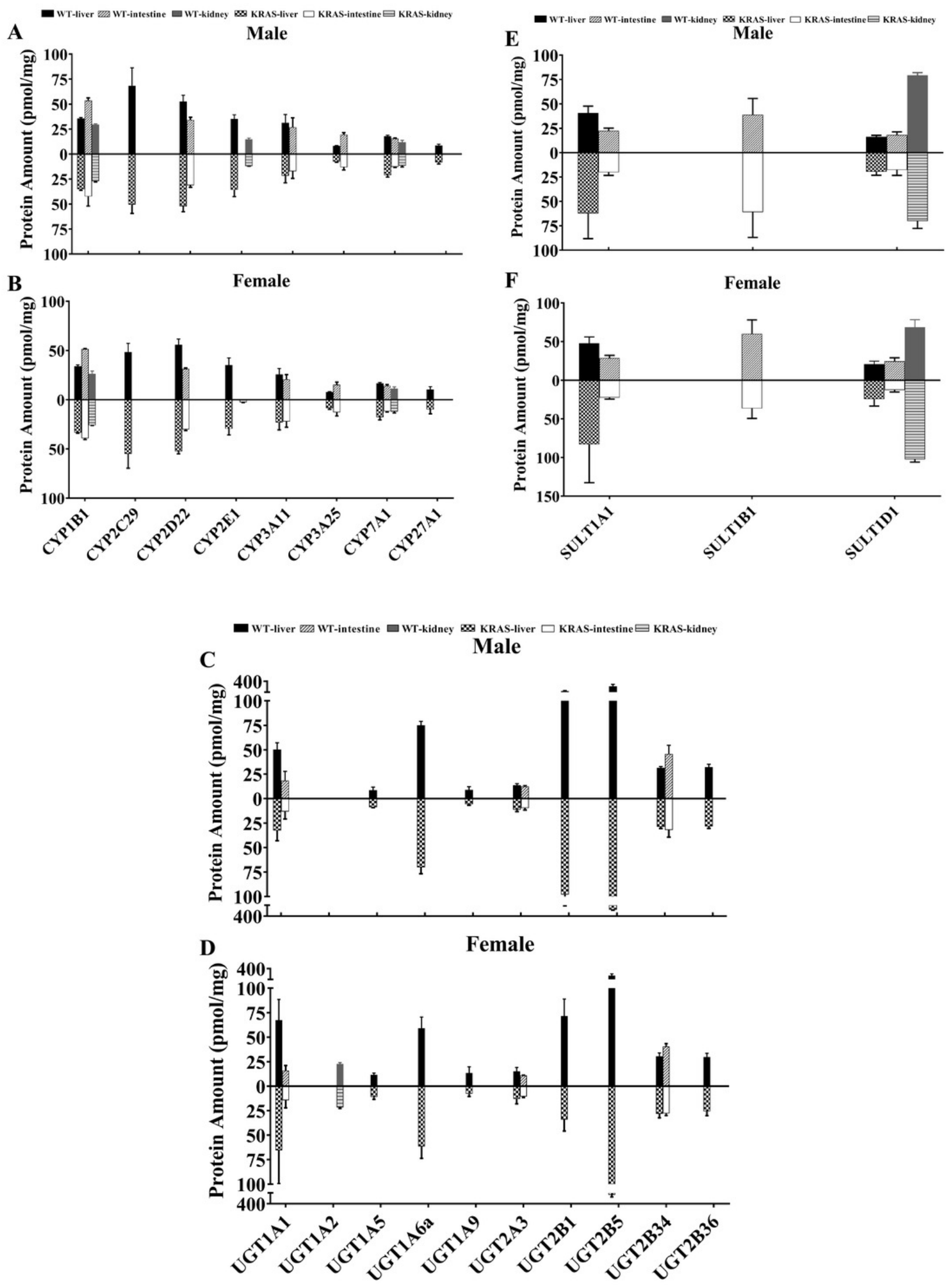


\section{Figure 7}

Alterations in protein levels of 8 CYPs, 9 UGTs and 2 SULTs at different ages in the liver of male KRAS and WT mice, $n=5$.

The dotted and solid lines represent the WT and KRAS mice, respectively. Each data point represents the mean \pm SD. The data were analyzed by one-way ANOVA (for normally distributed data) and Kruskal-Wallis $\mathrm{H}$ analysis (for non-normally distributed data). We adjusted the significance level $\alpha$ to 0.0125 according to the Bonferroni correction $(0.05 / 4=0.0125)$. The symbols " $A$ " and "a" indicate significant differences in the male WT and KRAS mice at 10,15, 20 and 26 weeks relative to 5 weeks, $p<0.0125$. 
.... Male WT
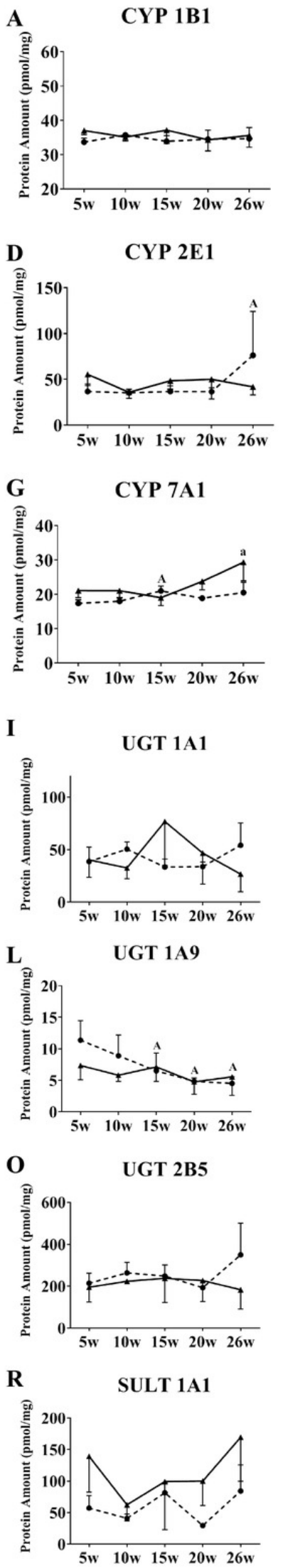
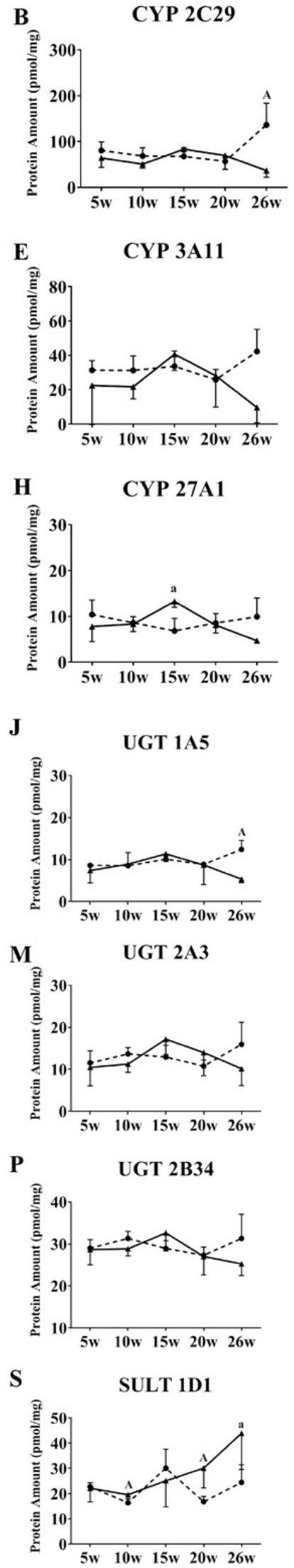

Male KRAS
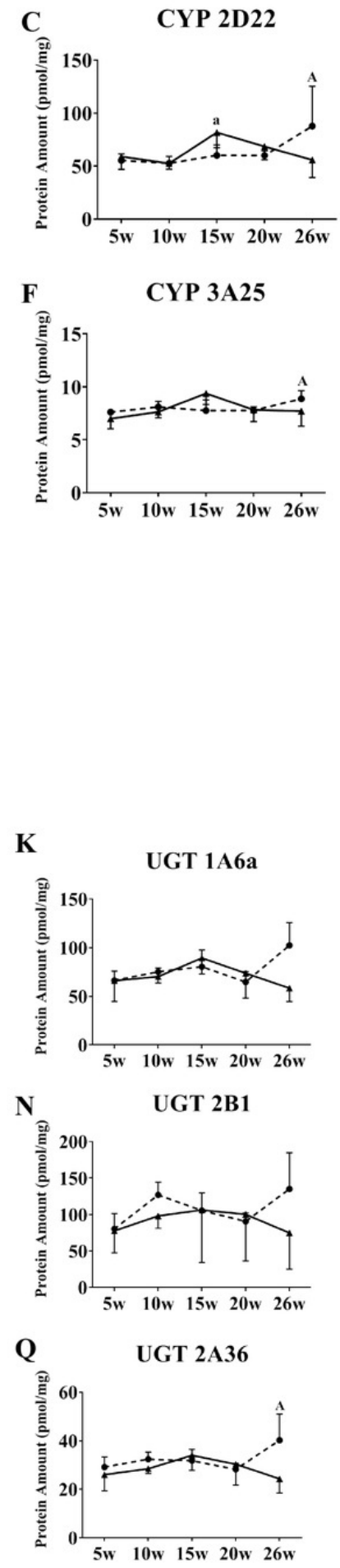


\section{Figure 8}

Alterations in protein levels of CYPs, UGTs and SULTs at different ages in the intestinal and kidney tissue of male KRAS and WT mice, $n=5$.

A-K shows the DMEs expression in intestinal tissue. L-O shows the DMEs expression in kidney tissue. The dotted and solid lines represent the WT and KRAS mice, respectively. Each data point represents the mean \pm SD. The data were analyzed by one-way ANOVA (for normally distributed data) and Kruskal-Wallis $\mathrm{H}$ analysis (for non-normally distributed data). We adjusted the significance level $\alpha$ to 0.0125 according to the Bonferroni correction $(0.05 / 4=0.0125)$. The symbols " $A$ " and " $a$ " indicate significant differences in the male WT and KRAS mice at 10, 15, 20 and 26 weeks relative to 5 weeks, $p<0.0125$. 
Male WT

B

A

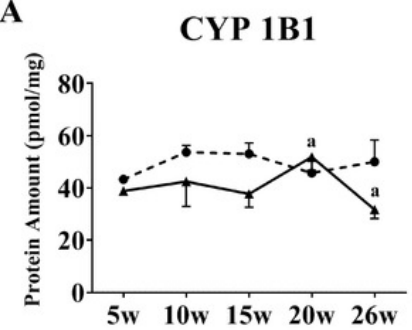

D

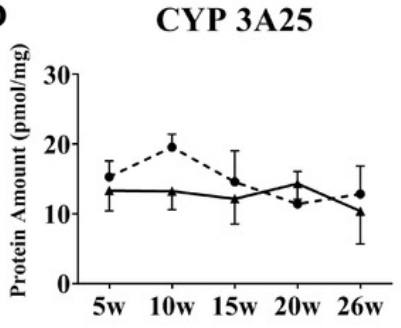

F
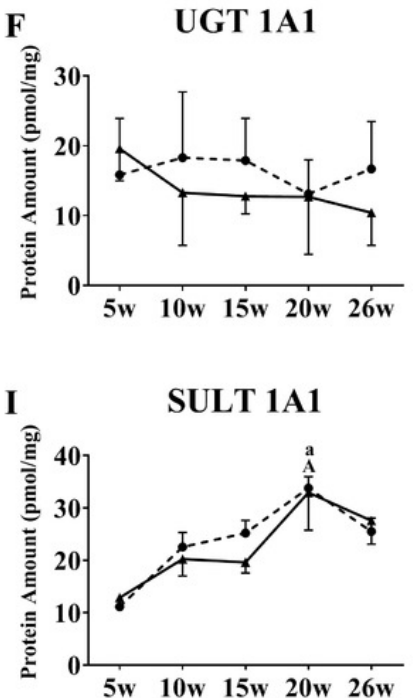

L

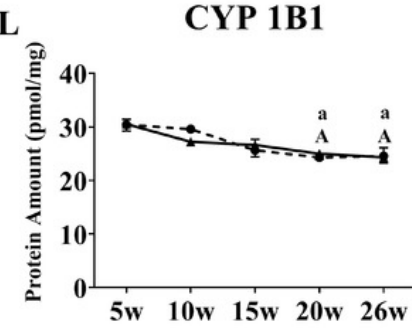

$\mathbf{N}$

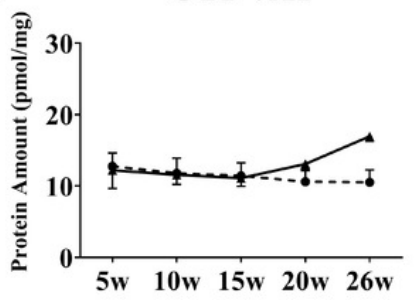

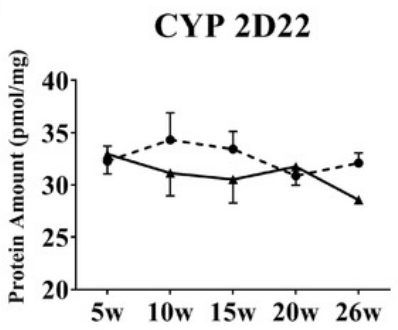

E

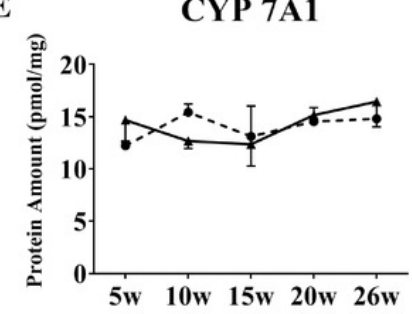

G

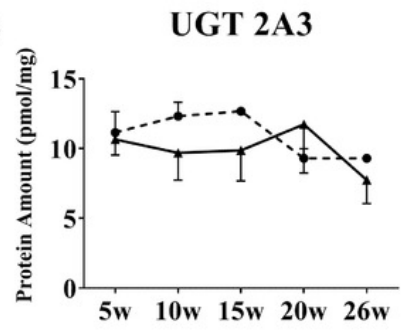

$\mathbf{J}$
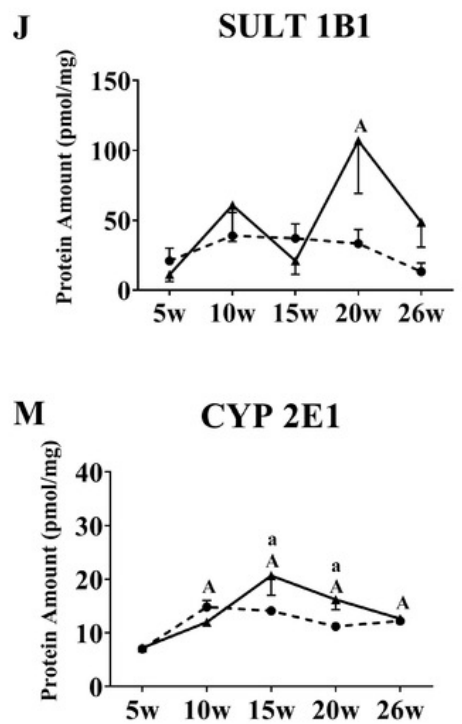

$\mathbf{O}$

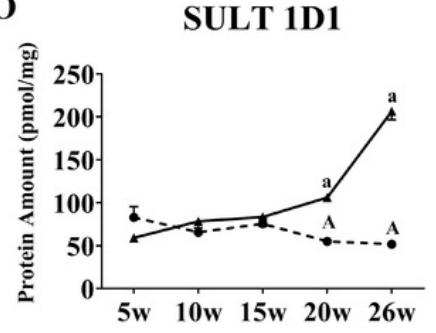

Male KRAS

C

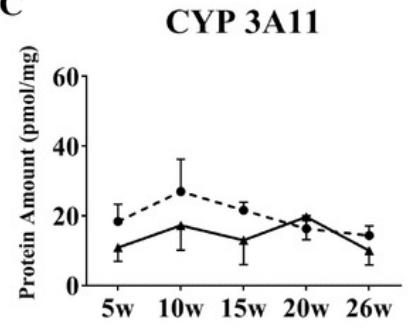

H

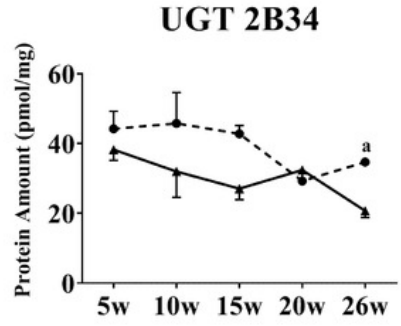

K

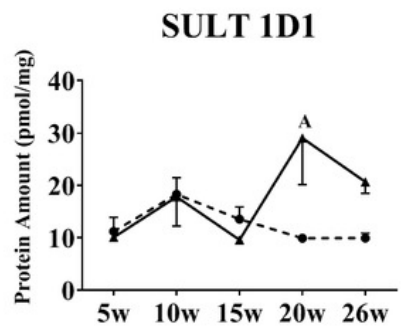

